\title{
Foundations of Boolean Stream Runtime Verification ${ }^{\star}$ (Extended Version)
}

\author{
Laura Bozzelli ${ }^{1}$ and César Sánchez ${ }^{2,3}$ \\ ${ }^{1}$ Technical University of Madrid (UPM), Madrid, Spain. \\ ${ }^{2}$ IMDEA Software Institute, Madrid, Spain \\ ${ }^{3}$ Institute for Information Security, CSIC, Spain.
}

\begin{abstract}
Stream runtime verification (SRV), pioneered by the tool LOLA, is a declarative approach to specify synchronous monitors.

In SRV, monitors are described by specifying dependencies between output streams of values and input streams of values. The declarative nature of SRV enables a separation between (1) the evaluation algorithms, and (2) the monitor storage and its individual updates. This separation allows SRV to be lifted from conventional failure monitors into richer domains to collect statistics of traces. Moreover, SRV allows to easily identify specifications that can be efficiently monitored online, and to generate efficient schedules for offline monitors.

In spite of these attractive features, many important theoretical problems about SRV are still open. In this paper, we address complexity, expressiveness, succinctness, and closure issues for the subclass of Boolean SRV (BSRV) specifications. Additionally, we show that for this subclass, offline monitoring can be performed with only two passes (one forward and one backward) over the input trace in spite of the alternation of past and future references in the BSRV specification.
\end{abstract}

\section{Introduction}

Runtime verification (RV) has emerged in the last decades as an applied formal technique for software reliability. In RV, a specification, expressing correctness requirements, is automatically translated into a monitor. Such a monitor is then used to check either the current execution of a running system, or a finite set of recorded executions with respect to the given specification. The former scenario is called online monitoring, while the latter one is called offline monitoring. Online monitoring is used to detect and possibly handle (e.g., by the execution of additional repair code) violations of the specification when the system is in operation. On the other hand, offline monitoring is used in post-mortem analysis and it is convenient for testing large systems before deployment. Unlike static verification (such as model-checking) which formally checks that all the (infinite)

* This work was funded in part by Spanish MINECO Project "TIN2012-39391-C04-01 STRONGSOFT" and by Spanish MINECO Project "TIN2012-38137-C02 VIVAC". 
executions or traces of a system satisfy the specification, RV only considers a single finite trace. Thus, this methodology sacrifices completeness guarantees to obtain an immediately applicable and formal extension of testing. See $[18,15]$ for modern surveys on runtime verification.

Stream runtime verification. The first specification formalisms proposed for runtime verification were based on specification languages for static verification, typically LTL [19] or past LTL adapted for finite paths $[16,9,5]$. Other formalisms for expressing monitors include regular expressions [24], rule based specifications as proposed in the logic Eagle [1], or rewriting [23]. Stream runtime verification (SRV), first proposed in the tool LOLA [8], is an alternative to define monitors for synchronous systems. In SRV, specifications declare explicitly the dependencies between input streams of values (representing the observable behavior of the system) and output streams of values (describing error reports and diagnosis information). These dependencies can relate the current value of an output stream with the values of the same or other streams in the present moment, in past instants (like in past temporal formulas) or in future instants. A similar approach to describe temporal relations as streams was later introduced as temporal testers [22].

Stream runtime verification offers two advantages to the description of monitors. First, SRV separates the algorithmic aspects of the runtime evaluation (by explicitly declaring the data dependencies) from the specific individual operations performed at each step (which depend on the type of data being observed, manipulated and stored). In this manner, well-known evaluation algorithms for monitoring Boolean observations - for example those from temporal logics - can be generalized to richer data domains, producing monitors that collect statistics about traces. Similarly to the Boolean case, the first approaches for collecting statistics from running traces were based on extensions of LTL [10]. SRV can be viewed as a generalization of these approaches to streams. Other modern approaches to the runtime verification for statistic collection extend firstorder LTL $[4,2,3]$. Moreover, the declarative nature of SRV allows to identify specifications that are amenable for efficient online monitoring, essentially those specifications whose values can be resolved by past and present observations. Additionally, the analysis of dependencies also allows to generate efficient offline monitors by scheduling passes over the dumped traces, where the number of passes (back and forth) depends on the number of alternations between past and future references in the specification.

SRV can be seen as a variation of synchronous languages [7] - like Esterel [6], Lustre [13] or Signal [11] - specifically designed for observing traces of systems, removing the causality assumption. In synchronous languages, stream values can only depend on past or present values, while in SRV a dependency on future values is additionally allowed to describe future temporal observations. In recent years, SRV has also been extended to real-time systems [21, 12].

When used for synthesizing monitors, SRV specifications need to be welldefined: for every input there is a unique corresponding output stream. However, as with many synchronous languages, the declarative style of SRV allows 
specifications that are not well-defined: for some observations, either there is no possible output (over-definedness) or there is more than one output (underdefinedness). This anomaly is caused by circular dependencies, and in [8], a syntactical constraint called well-formedness is introduced in order to ensure the absence of circular dependencies, and guarantee well-definedness.

Our contribution. In spite of its applicability, several foundational theoretical problems of SRV have not been studied so far. In this paper, we address complexity, expressiveness, succinctness, and closure properties for Boolean SRV (BSRV). Our results can be summarized as follows:

- we establish the complexity of checking whether a specification is underdefined, over-defined or well-defined. Apart from the theoretical significance of these results, many important practical properties of specifications (like semantic equivalence, implication and redundancy) can be reduced to the decision problems above.

- BSRV specifications can be naturally interpreted as language recognizers, where one selects the inputs for which the specification admits some output. We prove that in this setting, BSRV captures precisely the class of regular languages. We also show efficient closure constructions for many language operations. Additionally, BSRV specifications can be exponentially more succinct than nondeterministic finite-state automata (NFA).

- Finally, based on the construction of the NFA associated with a well-defined BSRV specification, we show how to schedule an offline algorithm with only two passes, one forward and one backward. This gives a partial answer (for the Boolean case) to the open problem of reducing the number of passes in offline monitoring for well-formed SRV specifications [8].

The rest of the paper is structured as follows. Section 2 revisits SRV. In Section 3 we establish expressiveness, succinctness, and closure results for BSRV specifications when interpreted as language recognizers. In Section 4, we describe the two-pass offline monitoring algorithm. Section 5 is devoted to the decision problems for BSRV specifications. Finally, Section 6 concludes. Due to lack of space, some proofs are omitted and are included in the appendix.

\section{Stream Runtime Verification (SRV)}

In this Section, we recall the SRV framework [8]. We focus on SRV specifications over stream variables of the same type (with emphasis on the Boolean type).

A type $T$ is a tuple $T=\langle D, F\rangle$ consisting of a countable value domain $D$ and a finite collection $F$ of interpreted function symbols $f$, where $f$ denotes a computable function from $D^{k}$ to $D$ and $k \geq 0$ is the specific arity of $f$. Note that 0 -ary function symbols (constants) are associated with individual values. In particular, we consider the Boolean type, where $D=\{0,1\}$ and $F$ consists of the Boolean operators $\wedge$ and $\vee$ and $\neg$. A stream of type $T$ is a non-empty finite word $w$ over the domain $D$ of $T$. Given such a stream $w,|w|$ is the length of $w$ and for all $1 \leq i \leq|w|, w(i)$ is the $i$ th letter of $w$ (the value of the stream at time step $i$ ). The stream $w$ is uniform if there is $d \in D$ such that $w$ is in $d^{*}$. 
For a finite set $Z$ of (stream) variables, a stream valuation of type $T$ over $Z$ is a mapping $\sigma$ assigning to each variable $z \in Z$, a stream $\sigma(z)$ of type $T$ such that the streams associated with the different variables in $Z$ have the same length $N$ for some $N \geq 1$. We also say that $N$ is the length of $\sigma$, which is denoted by $|\sigma|$.

Remark 1. Note that for the Boolean type, a stream valuation $\sigma$ over $Z$ can be identified with the non-empty word over $2^{Z}$ of length $|\sigma|$ whose $i$ th symbol, written $\sigma(i)$, is the set of variables $z \in Z$ such that $\sigma(z)(i)=1$.

Stream Expressions. Given a finite set $Z$ of variables, the set of stream expressions $E$ of type $T$ over $Z$ is inductively defined by the following syntax:

$$
\mathrm{E}:=\tau|\tau[\ell \mid c]| f\left(\mathrm{E}_{1}, \ldots, \mathrm{E}_{\mathrm{k}}\right)
$$

where $\tau$ is either a constant of type $T$ or a variable in $Z, \ell$ is a non-null integer, $c$ is a constant of type $T$, and $f \in F$ is a function of type $T$ and arity $k>0$. Informally, $\tau[\ell \mid c]$ refers to the value of $\tau$ offset $\ell$ positions from the current position, and the constant $c$ is the default value of type $T$ assigned to positions from which the offset is after the end or before the beginning of the stream. Stream expressions $\mathrm{E}$ of type $T$ over $Z$ are interpreted over stream valuations $\sigma$ of type $T$ over $Z$. The valuation of $\mathrm{E}$ with respect to $\sigma$, written $\llbracket \mathrm{E}, \sigma \rrbracket$, is the stream of type $T$ and length $|\sigma|$ inductively defined as follows for all $1 \leq i \leq|\sigma|$ :

$-\llbracket c, \sigma \rrbracket(i)=c$ and $\llbracket z, \sigma \rrbracket(i)=\sigma(z)(i)$ for all $z \in Z$

$-\llbracket \tau[\ell \mid c], \sigma \rrbracket(i)= \begin{cases}\llbracket \tau, \sigma \rrbracket(i+\ell) & \text { if } 1 \leq i+\ell \leq|\sigma| \\ c & \text { otherwise }\end{cases}$

$-\llbracket f\left(\mathrm{E}_{1}, \ldots, \mathrm{E}_{\mathrm{k}}\right), \sigma \rrbracket(i)=f\left(\llbracket \mathrm{E}_{1}, \sigma \rrbracket(i), \ldots, \llbracket \mathrm{E}_{\mathrm{k}}, \sigma \rrbracket(i)\right)$

For the Boolean type, we use some shortcuts: $\mathrm{E}_{1} \rightarrow \mathrm{E}_{2}$ stands for $\neg \mathrm{E}_{1} \vee \mathrm{E}_{2}$, $\mathrm{E}_{1} \leftrightarrow \mathrm{E}_{2}$ stands for $\left(\mathrm{E}_{1} \rightarrow \mathrm{E}_{2}\right) \wedge\left(\mathrm{E}_{2} \rightarrow \mathrm{E}_{1}\right)$, and if $\mathrm{E}$ then $\mathrm{E}_{1}$ else $\mathrm{E}_{2}$ stands for $\left(E \wedge E_{1}\right) \vee\left(\neg E \wedge E_{2}\right)$. Additionally, we use first and last for the Boolean stream expressions $0[-1 \mid 1]$ and $0[+1 \mid 1]$, respectively. Note that for a Boolean stream, first is 1 precisely at the first position, and last is 1 precisely at the last position.

Example 1. Consider the following Boolean stream expression E over $Z=\{x\}$ :

$$
\mathrm{E}:=\text { if } x \text { then } x \text { else } x[1 \mid 0]
$$

For every Boolean stream valuation $\sigma$ over $Z$ such that $\sigma(Z) \in(01)^{+}$, the valuation of $\mathrm{E}$ with respect to $\sigma$ is the uniform Boolean stream $1^{|\sigma|}$.

Stream Runtime Verification specification language (SRV). Given a finite set $X$ of input variables and a set $Y=\left\{y_{1}, \ldots, y_{n}\right\}$ of output variables with $X \cap Y=\emptyset$, an SRV $\varphi$ of type $T$ over $X$ and $Y$ is a set of equations

$$
\varphi:=\left\{y_{1}=\mathrm{E}_{1}, \ldots, y_{n}=\mathrm{E}_{\mathrm{n}}\right\}
$$

where $\mathrm{E}_{1}, \ldots, \mathrm{E}_{\mathrm{n}}$ are stream expressions of type $T$ over $X \cup Y$. Note that there is exactly one equation for each output variable. A stream valuation of $\varphi$ is a 
stream valuation of type $T$ over $X \cup Y$, while an input (resp., output) of $\varphi$ is a stream valuation of type $T$ over $X$ (resp., $Y$ ). Given an input $\sigma_{X}$ of $\varphi$ and an output $\sigma_{Y}$ of $\varphi$ such that $\sigma_{X}$ and $\sigma_{Y}$ have the same length, $\sigma_{X} \cup \sigma_{Y}$ denotes the stream valuation of $\varphi$ defined in the obvious way. The SRV $\varphi$ describes a relation, written $\llbracket \varphi \rrbracket$, between inputs $\sigma_{X}$ of $\varphi$ and outputs $\sigma_{Y}$ of $\varphi$, defined as follows: $\left(\sigma_{X}, \sigma_{Y}\right) \in \llbracket \varphi \rrbracket$ iff $\left|\sigma_{X}\right|=\left|\sigma_{Y}\right|$ and for each equation $y_{j}=\mathrm{E}_{\mathrm{j}}$ of $\varphi$,

$$
\llbracket y_{j}, \sigma \rrbracket=\llbracket \mathrm{E}_{\mathrm{j}}, \sigma \rrbracket \quad \text { where } \sigma=\sigma_{X} \cup \sigma_{Y}
$$

If $\left(\sigma_{X}, \sigma_{Y}\right) \in \llbracket \varphi \rrbracket$, we say that the stream valuation $\sigma_{X} \cup \sigma_{Y}$ is a valuation model of $\varphi$ (associated with the input $\sigma_{X}$ ). Note that in general, for a given input $\sigma_{X}$, there may be zero, one, or multiple valuation models associated with $\sigma_{X}$. This leads to the following notions for an $\operatorname{SRV} \varphi$ :

- Under-definedness: for some input $\sigma_{X}$, there are at least two distinct valuation models of $\varphi$ associated with $\sigma_{X}$.

- Over-definedness: for some input $\sigma_{X}$, there is no valuation model of $\varphi$ associated with $\sigma_{X}$.

- Well-definedness: for each input $\sigma_{X}$, there is exactly one valuation model of $\varphi$ associated with $\sigma_{X}$

Note that an SRV $\varphi$ may be both under-defined and over-defined, and $\varphi$ is well-defined iff it is neither under-defined nor over-defined. For runtime verification, SRV serves as a query language on program behaviors (input streams) from which one computes a unique answer (the output streams). In this context, a specification is useful only if it is well-defined. However, in practice, it is convenient to distinguish intermediate output variables from observable output variables separating output streams that are of interest to the user from those that are used only to facilitate the computation of other streams. This leads to a more general notion of well-definedness. Given a subset $Z \subseteq Y$ of output variables, an SRV $\varphi$ is well-defined with respect to $Z$ if for each input $\sigma_{X}$, there is exactly one stream valuation $\sigma_{Z}$ over $Z$ having the same length as $\sigma_{X}$ such that $\sigma_{X} \cup \sigma_{Z}$ can be extended to some valuation model of $\varphi$ (uniqueness of the output streams over $Z$ ).

Analogously, we consider a notion of semantic equivalence between SRV of the same type and having the same input variables, which is parameterized by a set of output variables. Formally, given an SRV $\varphi$ of type $T$ over $X$ and $Y$, an SRV $\varphi^{\prime}$ of type $T$ over $X$ and $Y^{\prime}$, and $Z \subseteq Y \cap Y^{\prime}$, we say that $\varphi$ and $\varphi^{\prime}$ are equivalent with respect to $Z$ if for each valuation model $\sigma$ of $\varphi$, there is a valuation model $\sigma^{\prime}$ of $\varphi^{\prime}$ such that $\sigma$ and $\sigma^{\prime}$ coincide on $X \cup Z$, and vice versa. Moreover, if $Y^{\prime} \supseteq Y$, then we say that $\varphi^{\prime}$ is $\varphi$-equivalent if $\varphi$ and $\varphi^{\prime}$ are equivalent with respect to $Y$.

Remark 2. In the rest of the paper, we focus on Boolean SRV (BSRV for short). Thus, in the following, we omit the reference to the type $T$ in the various definitions. We assume that the offsets $\ell$ in the subexpressions $\tau[\ell \mid c]$ of a BSRV are encoded in unary. For a Boolean stream expression $E$, we denote by $\|E\|$ the 
offset $\ell$ if $E$ is a stream expression of the form $\tau[\ell \mid c]$; otherwise, $\|E\|$ is 1 . The size $|\varphi|$ of a $\operatorname{BSRV} \varphi$ is defined as $|\varphi|:=\sum_{\mathrm{E} \in S E(\varphi)}\|\mathrm{E}\|$, where $S E(\varphi)$ is the set of stream subexpressions of $\varphi$.

Example 2. Consider the following BSRV over $X=\{x\}$ and $Y=\{y\}$ :

$\varphi_{1}:=\{y=x \wedge y\} \quad \varphi_{2}:=\{y=x \wedge \neg y\} \quad \varphi_{3}:=\{y=$ if $x$ then $x[2 \mid 0]$ else $x[-2 \mid 0]\}$

The specification $\varphi_{1}$ is under-defined since $\left(1^{N}, 0^{N}\right)$ and $\left(1^{N}, 1^{N}\right)$ are two valuation models for each $N \geq 1$. On the other hand, the specification $\varphi_{2}$ is overdefined since for each $N \geq 1$, there is no valuation model associated with the input $1^{N}$. Finally, the specification $\varphi_{3}$ is well-defined.

\section{BSRV as Language Recognizers}

BSRV can be interpreted as a simple declarative formalism to specify languages of non-empty finite words. Formally, we associate to a BSRV $\varphi$ over $X$ and $Y$, the language $\mathcal{L}(\varphi)$ of non-empty finite words over $2^{X}$ (or, equivalently, input stream valuations) for which the specification $\varphi$ admits a valuation model, i.e.,

$$
\mathcal{L}(\varphi):=\left\{\sigma_{X} \mid\left(\sigma_{X}, \sigma_{Y}\right) \in \llbracket \varphi \rrbracket \text { for some } \sigma_{Y}\right\}
$$

Example 3. Let $X=\{x\}, Y=\{y\}$, and $\varphi=\{y=$ if E then $y$ else $\neg y\}$, where

$$
\mathrm{E}:=(\text { first } \rightarrow(x \wedge y)) \wedge(y \rightarrow \neg y[+1 \mid 0]) \wedge(\neg y \rightarrow(x[+1 \mid 1] \wedge y[+1 \mid 1]))
$$

A pair $\left(\sigma_{X}, \sigma_{Y}\right)$ is a valuation model of $\varphi$ iff the valuation of the stream expression $\mathrm{E}$ w.r.t. $\sigma_{X} \cup \sigma_{Y}$ is in $1^{+}$iff $\sigma_{X}(x)(i)=1$ for all odd positions $i$. Hence, $\mathcal{L}(\varphi)$ is the set of Boolean streams which assume the value 1 at the odd positions.

In the following, we show that BSRV, as language recognizers, are effectively equivalent to nondeterministic finite automata (NFA) on finite words. While the translation from NFA to BSRV can be done in polynomial time, the converse translation involves an unavoidable singly exponential blowup. Moreover, BSRV turn out to be effectively and efficiently closed under many language operations.

In order to present our results, we shortly recall the class of NFA on finite words. An NFA $\mathcal{A}$ over a finite input alphabet $I$ is a tuple $\mathcal{A}=\left\langle Q, q_{0}, \delta, F\right\rangle$, where $Q$ is a finite set of states, $q_{0} \in Q$ is the initial state, $\delta: Q \times I \rightarrow 2^{Q}$ is the transition function, and $F \subseteq Q$ is a set of accepting states. Given an input word $w \in I^{*}$, a run $\pi$ of $\mathcal{A}$ over $w$ is a sequence of states $\pi=q_{1}, \ldots, q_{|w|+1}$ such that $q_{1}$ is the initial state and for all $1 \leq i \leq|w|, q_{i+1} \in \delta\left(q_{i}, w(i)\right)$. The run $\pi$ is accepting if it leads to an accepting state (i.e, $q_{|w|+1} \in F$ ). The language $\mathcal{L}(\mathcal{A})$ accepted by $\mathcal{A}$ is the set of non-empty finite words $w$ over $I$ such that there is an accepting run of $\mathcal{A}$ over $w$. $\mathcal{A}$ is universal if $\mathcal{L}(\mathcal{A})=I^{+}$. A language over non-empty finite words is regular if it is accepted by some NFA. An NFA is unambiguous if for each input word $w$, there is at most one accepting run on $w$. 
Fix a BSRV $\varphi$ on $X$ and $Y$. In order to build an NFA accepting $\mathcal{L}(\varphi)$, we define an encoding of the valuation models of $\varphi$. For this, we associate to $\varphi$ two parameters, theback reference distance $b(\varphi)$ and theforward reference distance $f(\varphi)$ :

$b(\varphi):=\max (0,\{\ell \mid \ell>0$ and $\varphi$ contains a subexpression of the form $z[-\ell, c]\})$ $f(\varphi):=\max (0,\{\ell \mid \ell>0$ and $\varphi$ contains a subexpression of the form $z[\ell, c]\})$

For a stream valuation $\sigma$ of $\varphi$ and an expression $\mathrm{E}$ of $\varphi$, the value of $\mathrm{E}$ w.r.t. $\sigma$ at a time step $i$ is completely specified by the values of $\sigma$ at time steps $j$ such that $i-b(\varphi) \leq j \leq i+f(\varphi)$. We define the following alphabets:

$$
A:=2^{X \cup Y} \quad A_{\perp}:=A \cup\{\perp\} \quad P_{\varphi}:=\left(A_{\perp}\right)^{b(\varphi)} \times A \times\left(A_{\perp}\right)^{f(\varphi)}
$$

where $\perp$ is a special symbol. Note that a stream valuation of $\varphi$ corresponds to a non-empty finite word over the alphabet $A$, and the cardinality of $P_{\varphi}$ is singly exponential in the size of $\varphi$. For an element $p=\left(a_{-b(\varphi)}, \ldots, a_{-1}, a_{0}, a_{1}, \ldots, a_{f(\varphi)}\right)$ of $P_{\varphi}$, the component $a_{0}$, called the main value of $p$, intuitively represents the value of some stream valuation $\sigma$ at some time step $i$, while $a_{-b(\varphi)}, \ldots, a_{-1}$ (resp., $\left.a_{1}, \ldots, a_{f(\varphi)}\right)$ represent the values of $\sigma$ at the previous $b(\varphi)$ (resp., next $f(\varphi))$ time steps, if any (the symbol $\perp$ is used to denote the absence of a previous or next time step). Let $\tau$ be either a Boolean constant or a variable in $X \cup Y$, and $a \in A$. Then, the Boolean value of $\tau$ in $a$ is $\tau$ if $\tau$ is a constant, otherwise the value is 1 iff $\tau \in a$. For a Boolean stream expression $\mathrm{E}$ over $X \cup Y$ and an element $p=\left(a_{-b(\varphi)}, \ldots, a_{-1}, a_{0}, a_{1}, \ldots, a_{f(\varphi)}\right)$ of $P_{\varphi}$, the value $\llbracket E, p \rrbracket$ of $E$ with respect to $p$ is the computable Boolean value inductively defined as follows:

$-\llbracket c, p \rrbracket=c$ and $\llbracket z, p \rrbracket=$ the value of $z$ in $a_{0}$

$-\llbracket \tau[\ell \mid c], p \rrbracket= \begin{cases}\text { the value of } \tau \text { in } a_{\ell} & \text { if }-b(\varphi) \leq \ell \leq f(\varphi) \text { and } a_{\ell} \neq \perp \\ c & \text { otherwise }\end{cases}$

$-\llbracket f\left(\mathrm{E}_{1}, \ldots, \mathrm{E}_{\mathrm{k}}\right), p \rrbracket=f\left(\llbracket \mathrm{E}_{1}, p \rrbracket, \ldots, \llbracket \mathrm{E}_{\mathrm{k}}, p \rrbracket\right)$

We denote by $Q_{\varphi}$ the subset of $P_{\varphi}$ consisting of the elements $p$ of $P_{\varphi}$ such that for each equation $y=\mathrm{E}$ of $\varphi$, the value of $y$ with respect to $p$ coincides with the value of $\mathrm{E}$ with respect to $p$. Let \# be an additional special symbol (which will be used as initial state of the NFA associated with $\varphi$ ). An expanded valuation model of $\varphi$ is a word of the form \# $w$ such that $w$ is a non-empty finite word $w$ over the alphabet $Q_{\varphi}$ satisfying the following:

- $w(1)$ is of the form $\left(\perp, \ldots, \perp, a_{0}, a_{1}, \ldots, a_{f(\varphi)}\right)$;

$-w(|w|)$ is of the form $\left(a_{-b(\varphi)}, \ldots, a_{-1}, a_{0}, \perp, \ldots, \perp\right)$;

- if $1 \leq i<|w|$ and $w(i)=\left(a_{-b(\varphi)}, \ldots, a_{-1}, a_{0}, a_{1}, \ldots, a_{f(\varphi)}\right)$, then there is $d \in$ $A_{\perp}$ such that $w(i+1)$ is of the form $\left(a_{-b(\varphi)+1}, \ldots, a_{-1}, a_{0}, a_{1}, \ldots, a_{f(\varphi)}, d\right)$.

For an expanded valuation model \# $\cdot w$ of $\varphi$, the associated stream valuation $\sigma(w)$ is the stream valuation of $\varphi$ of length $|w|$ whose $i$ th element is the main value of the $i$ th element of $w$. By construction, we easily obtain that $\sigma(w)$ is a valuation model of $\varphi$ and, more precisely, the following lemma holds. 
Lemma 1. The mapping assigning to each expanded valuation model $\# \cdot w$ of $\varphi$ the associated stream valuation $\sigma(w)$ is a bijection between the set of expanded valuation models of $\varphi$ and the set of valuation models of $\varphi$.

By the above characterization of the set of valuations models of a BSRV $\varphi$, we easily obtain the following result.

Theorem 1 (From BSRV to NFA). Given a BSRV $\varphi$ over $X$ and $Y$, one can construct in singly exponential time an NFA $\mathcal{A}_{\varphi}$ over the alphabet $2^{X}$ accepting $\mathcal{L}(\varphi)$ whose set of states is $Q_{\varphi} \cup\{\#\}$. Moreover, for each input $\sigma_{X}$, the set of accepting runs of $\mathcal{A}_{\varphi}$ over $\sigma_{X}$ is the set of expanded valuation models of $\varphi$ encoding the valuation models of $\varphi$ associated with the input $\sigma_{X}$.

Proof. The NFA $\mathcal{A}_{\varphi}$ is defined as $\mathcal{A}_{\varphi}=\left\langle Q_{\varphi} \cup\{\#\}, \#, \delta_{\varphi}, F_{\varphi}\right\rangle$, where $F_{\varphi}$ is the set of elements of $Q_{\varphi}$ of the form $\left(a_{-b(\varphi)}, \ldots, a_{-1}, a_{0}, \perp, \ldots, \perp\right)$, and $\delta(p, \iota)$ is defined as follows for all states $p$ and input symbol $\iota \in 2^{X}$ :

- if $p=\#$, then $\delta_{\varphi}(p, \iota)$ is the set of states of the form $\left(\perp, \ldots, \perp, a_{0}, a_{1}, \ldots, a_{f(\varphi)}\right)$ such that $a_{0} \cap X=\iota$;

- if $p=\left(a_{-b(\varphi)}, \ldots, a_{-1}, a_{0}, a_{1}, \ldots, a_{f(\varphi)}\right) \in Q_{\varphi}$, then $\delta_{\varphi}(p, \iota)$ is the set of states of the form $\left(a_{-b(\varphi)+1}, \ldots, a_{-1}, a_{0}, a_{1}, \ldots, a_{f(\varphi)}, d\right)$ for some $d \in A_{\perp}$ whose main value $a$ satisfies $a \cap X=\iota$.

By construction, for each input $\sigma_{X}$, the set of accepting runs of $\mathcal{A}_{\varphi}$ over $\sigma_{X}$ coincides with the set of expanded valuation models \# $w$ of $\varphi$ such that the stream valuation $\sigma(w)$ is associated with the input $\sigma_{X}$. Thus, by Lemma 1 , the result follows.

For the converse translation from NFA to BSRV, we show the following.

Theorem 2 (From NFA to BSRV). Given an NFA $\mathcal{A}$ over the input alphabet $2^{X}$, one can construct in polynomial time a $B S R V \varphi_{\mathcal{A}}$ with set of input variables $X$ such that $\mathcal{L}\left(\varphi_{\mathcal{A}}\right)=\mathcal{L}(\mathcal{A})$.

Proof. Let $\mathcal{A}=\left\langle Q, q_{0}, \delta, F\right\rangle$. We construct a BSRV $\varphi_{\mathcal{A}}$ over the set of input variables $X$ as follows. First, for each input symbol $\iota$, we use a Boolean expression $\mathrm{E}_{\iota}$ over $X$, encoding the input symbol $\iota$, defined as $\mathrm{E}_{\iota}:=\left(\bigwedge_{x \in \iota} x\right) \wedge\left(\bigwedge_{x \in X \backslash \iota} \neg x\right)$. The set $Y$ of output variables of $\varphi_{\mathcal{A}}$ is defined as follows:

$$
Y=\bigcup_{q \in Q}\{\mathbf{q}\} \cup\{\text { control }\}
$$

Thus, we associate to each state $q \in Q$, an output variable $\mathrm{q}$, whose associated equation is the trivial one given by $\mathrm{q}=\mathrm{q}$. The equation for the output variable control is given by

$$
\text { control }=\text { if } \mathrm{E}_{\mathrm{ev}} \text { then control else } \neg \text { control }
$$


where the boolean stream expression $E_{\mathrm{ev}}$ describes accepting runs of the NFA $\mathcal{A}$ and is defined as follows:

$$
\begin{aligned}
& \mathrm{E}_{\mathrm{ev}}=\underbrace{\bigvee_{q \in Q}\left(\mathrm{q} \wedge \bigwedge_{p \in Q \backslash\{q\}} \neg \mathrm{p}\right)}_{\text {at each step, } \mathcal{A} \text { is exactly in one state }} \wedge \underbrace{\left(\text { first } \longrightarrow \mathrm{q}_{0}\right)}_{\text {a run of } \mathcal{\mathcal { A } \text { starts at the initial state }}} \wedge \\
& \underbrace{\bigwedge_{q \in Q} \bigwedge_{\iota \in I}\left(\left(\mathrm{q} \wedge \mathrm{E}_{\iota}\right) \longrightarrow \bigvee_{p \in \delta(q, \iota)} \mathrm{p}[+1 \mid 1]\right)}_{\text {the evolution of } \mathcal{A} \text { is } \delta \text {-consistent }} \wedge \underbrace{\left(\text { last } \longrightarrow \bigvee_{(q, \iota) \in\{(q, \iota) \mid \delta(q, \iota) \cap F \neq \emptyset)\}}\left(\mathrm{q} \wedge \mathrm{E}_{\iota}\right)\right)}_{\text {the run of } \mathcal{A} \text { is accepting }}
\end{aligned}
$$

By construction, it easily follows that given an input stream valuation $\sigma_{X}$, there is a valuation model of $\varphi_{\mathcal{A}}$ associated with the input $\sigma_{X}$ if and only if there is a stream valuation $\sigma$ associated with the input $\sigma_{X}$ such that the valuation of $\mathrm{E}_{\mathrm{ev}}$ with respect to $\sigma$ is a uniform stream in $1^{+}$if and only if there is an accepting run of $\mathcal{A}$ over the input $\sigma_{X}$. Hence, the result follows.

As a corollary of Theorems 1 and 2, we obtain the following result.

Corollary 1. BSRV, when interpreted as language recognizers, capture the class of regular languages over non-empty finite words.

Succinctness issues. It turns out that the singly exponential blow-up in Theorem 1 cannot be avoided. To prove this we first show a linear time translation from standard linear temporal logic LTL with past over finite words (which captures a subclass of regular languages) into BSRV. Recall that formulas $\psi$ of LTL with past over a finite set $A P$ of atomic propositions are defined as follows:

$$
\psi:=p|\neg \psi| \psi \vee \psi|\bigcirc \psi| \ominus \psi|\psi \mathcal{U} \psi| \psi \mathcal{S} \psi
$$

where $p \in A P$ and $\bigcirc, \Theta, \mathcal{U}$, and $\mathcal{S}$ are the 'next', 'previous', 'until', and 'since' temporal modalities. For a finite word $w$ over $2^{A P}$ and a position $1 \leq i \leq|w|$, the satisfaction relation $(w, i) \models \psi$ is defined as follows (we omit the rules for the boolean connectives and the atomic propositions, which are standard):

$$
\begin{array}{ll}
(w, i) \models \bigcirc \psi & \Leftrightarrow i+1 \leq|w| \text { and }(w, i+1) \models \psi \\
(w, i) \models \ominus \psi & \Leftrightarrow i>1 \text { and }(w, i-1) \models \psi \\
(w, i) \models \psi_{1} \mathcal{U} \psi_{2} \Leftrightarrow \exists i \leq j \leq|w|,(w, j) \models \psi_{2} & \Leftrightarrow \text { and } \forall i \leq h<j,(w, h) \models \psi_{1} \\
(w, i) \models \psi_{1} \mathcal{S} \psi_{2} \Leftrightarrow \exists 1 \leq j \leq i,(w, j) \models \psi_{2} & \Leftrightarrow \text { and } \forall j<h \leq i,(w, h) \models \psi_{1}
\end{array}
$$

The language $\mathcal{L}(\psi)$ of a LTL formula $\psi$ is the set of non-empty finite words $w$ over $2^{A P}$ such that $(w, 1) \models \psi$.

Proposition 1. LTL with past can be translated in linear time into BSRV.

Proof. Let $\psi$ be a formula of LTL with past over a finite set $A P$ of atomic propositions. We construct in linear time a BSRV specification $\varphi$ over the set 
of input variables $X=A P$ such that $\mathcal{L}(\varphi)=\mathcal{L}(\psi)$. Let $S F(\psi)$ be the set of subformulas of $\psi$. Then, the set of output variables $Y$ of $\varphi$ is defined as follows.

$$
Y=\bigcup_{\theta \in S F(\psi)}\left\{y_{\theta}\right\} \cup\{\text { init }\}
$$

Thus, we associate to each subformula $\theta$ of $\psi$, an output variable $y_{\theta}$. The intended meaning is that for an input valuation $\sigma_{X}$ (corresponding to a non-empty finite word over $2^{A P}$ ) and a valuation model $\sigma$ associated with $\sigma_{X}$, at each time step $i$, the value of variable $y_{\theta}$ is 1 iff $\theta$ holds at position $i$ along $\sigma_{X}$. The equations for the output variables are defined as follows, where $p \in A P=X$.

$$
\begin{array}{ll}
\text { init }=\text { first } \rightarrow\left(y_{\psi} \vee \neg \text { init }\right) & y_{p}=p \\
y_{\neg \theta}=\neg y_{\theta} & y_{\theta_{1} \vee \theta_{2}}=y_{\theta_{1}} \vee y_{\theta_{2}} \\
y_{\bigcirc \theta}=y_{\theta}[+1 \mid 0] & y_{\ominus \theta}=y_{\theta}[-1 \mid 0] \\
y_{\theta_{1} \mathcal{U} \theta_{2}}=y_{\theta_{2}} \vee\left(\neg \text { last } \wedge y_{\theta_{1}} \wedge y_{\theta_{1} \mathcal{U} \theta_{2}}[+1 \mid 1]\right) & \\
y_{\theta_{1} \mathcal{S}} \mathcal{S} \theta_{2}=y_{\theta_{2}} \vee\left(\neg \text { first } \wedge y_{\theta_{1}} \wedge y_{\theta_{1}} \mathcal{S} \theta_{2}[-1 \mid 1]\right) &
\end{array}
$$

We need to show that $\mathcal{L}(\varphi)=\mathcal{L}(\psi)$. We prove the two set inclusions separately:

- For the inclusion $\mathcal{L}(\varphi) \subseteq \mathcal{L}(\psi)$, let $\sigma_{X} \in \mathcal{L}(\varphi)$. Hence, there is a valuation model $\sigma$ of $\varphi$ associated with the input $\sigma_{X}$. We need to show that $\left(\sigma_{X}, 1\right)=$ $\psi$. By construction and structural induction, for all $\theta \in S F(\psi)$ and positions $i$ along $\sigma_{X},\left(\sigma_{X}, i\right) \models \theta$ if and only if $\sigma\left(y_{\theta}\right)[i]=1$. Moreover, the equation for the output variable init ensures that $\sigma\left(y_{\psi}\right)[1]=1$. Hence, $\left(\sigma_{X}, 1\right) \models \psi$, and we are done.

- For the converse inclusion $\mathcal{L}(\psi) \subseteq \mathcal{L}(\varphi)$, let $\sigma_{X} \in \mathcal{L}(\psi)$, hence, $\left(\sigma_{X}, 1\right)=\psi$. We define a stream valuation $\sigma$ associated with the input $\sigma_{X}$ as follows: $\sigma($ init $)=1^{|\sigma|}$ and for all $\theta \in S F(\psi)$ and positions $i$ along $\sigma_{X}, \sigma\left(y_{\theta}\right)[i]=1$ if $\left(\sigma_{X}, i\right) \models \theta$, and $\sigma\left(y_{\theta}\right)[i]=0$ otherwise.

Since $\left(\sigma_{X}, 1\right) \models \psi$, by construction, it easily follows that $\sigma$ is a valuation model of $\varphi$ associated with the input $\sigma_{X}$. Hence, $\sigma_{X} \in \mathcal{L}(\varphi)$.

This concludes the proof.

It is well-known that there is a singly exponential succinctness gap between LTL with past and NFA [17]. Consequently, we obtain the following result.

Theorem 3. BSRV are singly exponentially more succinct than NFA, that is, there is a finite set $X$ of input variables and a family $\left(\varphi_{n}\right)_{n \geq 1}$ of BSRV such that for all $n \geq 1, \varphi_{n}$ has input variables in $X$ and size polynomial in $n$, and every NFA accepting $\mathcal{L}\left(\varphi_{n}\right)$ has at least $2^{\Omega(n)}$ states.

Effective closure under language operations. An interesting feature of the class of BSRV is that, when interpreted as language recognizers, BSRV are effectively and efficiently closed under many language operations. For two languages $\mathcal{L}$ and $\mathcal{L}^{\prime}$ of finite words, $\mathcal{L}^{R}$ denotes the reversal of $\mathcal{L}, \mathcal{L} \cdot \mathcal{L}^{\prime}$ denotes the concatenation of $\mathcal{L}$ and $\mathcal{L}^{\prime}$, and $\mathcal{L}^{+}$denotes the positive Kleene closure of $\mathcal{L}$. 


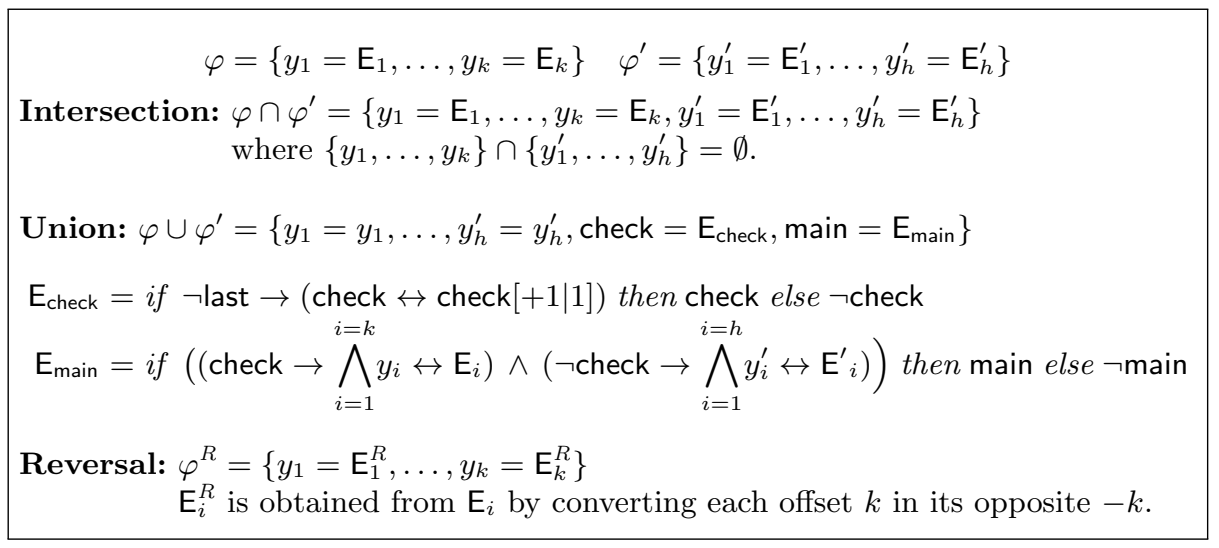

Fig. 1. Constructions for intersection, union, and reversal.

For a BSRV $\varphi$, we say that an output variable $y$ of $\varphi$ is uniform if for each valuation model of $\varphi$, the stream for $y$ is uniform.

Theorem 4. BSRV are effectively closed under the following language operations: intersection, union, reversal, positive Kleene closure, and concatenation. Additionally, the constructions for these operations can be done in linear time.

Proof. We illustrate the constructions for the considered language operations.

Details on their correctness of these constructions can be found in Appendix A.1.

Intersection, Union, and Reversal. The constructions are illustrated in Fig. 1. For the intersection, assuming w.l.o.g. that the BSRV $\varphi$ and $\varphi^{\prime}$ have no output variable in common, the BSRV recognizing $\mathcal{L}(\varphi) \cap \mathcal{L}\left(\varphi^{\prime}\right)$ is simply the joint set of the equations of $\varphi$ and $\varphi^{\prime}$. For the union, we use two new output variables check and main. Intuitively, check is a uniform output variable used to guess whether the input has to be considered an input for $\varphi$ or for $\varphi^{\prime}$. The equation for check ensures that the streams for check range over all the uniform Boolean streams. Depending on the uniform value of check (if it is in $0^{+}$or $1^{+}$), the equation for the output variable main ensures that the input is recognized iff either the equations of $\varphi$ are fulfilled or the equations of $\varphi^{\prime}$ are fulfilled. For the reversal, the BSRV recognizing $\mathcal{L}(\varphi)^{R}$ is obtained from $\varphi$ by replacing each subexpression $\tau[k \mid d]$ (resp., $\tau[-k \mid d]$ ) with $k>0$ with the subexpression $\tau[-k \mid d]$ (resp., $\tau[k \mid d]$ ). Positive Kleene closure. The construction is given in Fig. 2.

The BSRV recognizing $[\mathcal{L}(\varphi)]^{+}$uses two new output variables: wbegin and wend. Intuitively, wbegin and wend are used for guessing a decomposition in the given input $\sigma_{X}$ of the form $\sigma_{X}=\sigma_{X, 1} \cdot \ldots \cdot \sigma_{X, N}$ for some $N \geq 1$ in such a way that each component $\sigma_{X, i}$ is in $\mathcal{L}(\varphi)$. In particular, the output variable wbegin (resp., wend) is used to mark the first (resp., the last) positions of the components $\sigma_{X, i}$. Moreover, the equations for the output variables of $\varphi$ are modified to allow checking for an offset $k$ of $\varphi$ and a position $j$ inside a component $\sigma_{X, i}$ in the 
Positive Kleene closure for $\varphi=\left\{y_{1}=\mathrm{E}_{1}, \ldots, y_{k}=\mathrm{E}_{k}\right\}$

$$
\begin{gathered}
\varphi^{+}=\left\{y_{1}=\mathrm{E}_{1}^{+}, \ldots, y_{k}=\mathrm{E}_{k}^{+}, \text {wbegin }=\mathrm{E}_{\text {wbegin }}, \text { wend }=\mathrm{E}_{\text {wend }}\right\} \\
\mathrm{E}_{\text {wbegin }}=\text { if }(\text { first } \rightarrow \text { wbegin }) \wedge(\text { wbegin } \rightarrow \text { wend }[-1 \mid 1]) \text { then } \text { wbegin else } \neg \text { wbegin } \\
\mathrm{E}_{\text {wend }}=\text { if }(\text { last } \rightarrow \text { wend }) \wedge(\text { wend } \rightarrow \text { wbegin }[+1 \mid 1]) \text { then } \text { wend } \text { else } \neg \text { wend }
\end{gathered}
$$

and $\mathrm{E}_{i}^{+}$is obtained from $\mathrm{E}_{i}$ by replacing each stream subexpression $\tau[k \mid d]$ with $\mathrm{E}_{\tau, k, d}$ :

$$
\mathrm{E}_{\tau, k, d}= \begin{cases}\text { if } \bigvee_{\substack{j=1 \\ j=-k}} \text { wbegin }[j \mid 1] \text { then d else } \tau[k \mid d] \quad \text { if } k>0 \\ \text { if } \bigvee_{j=1} \text { wend }[-j \mid 1] \text { then d else } \tau[k \mid d] \text { if } k<0\end{cases}
$$

Fig. 2. Construction for positive Kleene closure

$$
\varphi=\left\{y_{1}=\mathrm{E}_{1}, \ldots, y_{k}=\mathrm{E}_{k}\right\} \quad \varphi^{\prime}=\left\{y_{1}^{\prime}=\mathrm{E}_{1}^{\prime}, \ldots, y_{h}^{\prime}=\mathrm{E}_{h}^{\prime}\right\}
$$

Concatenation: $\left\{y_{1}, \ldots, y_{k}\right\} \cap\left\{y_{1}^{\prime}, \ldots, y_{h}^{\prime}\right\}=\emptyset$

$\varphi \cdot \varphi^{\prime}=\left\{y_{1}=\right.$ if wmark then $\widetilde{\mathrm{E}}_{1}$ else $y_{1}, \ldots, y_{k}=$ if wmark then $\widetilde{\mathrm{E}}_{k}$ else $y_{k}$, $y_{1}^{\prime}=$ if $\neg$ wmark then $\widetilde{\mathrm{E}}_{1}^{\prime}$ else $y_{1}^{\prime}, \ldots, y_{h}^{\prime}=$ if $\neg$ wmark then $\widetilde{\mathrm{E}}_{h}^{\prime}$ else $y_{h}^{\prime}$, wmark $\left.=\mathrm{E}_{\text {wmark }}\right\}$

$$
\begin{gathered}
\mathrm{E}_{\mathrm{wmark}}=\text { if }(\text { first } \rightarrow \text { wmark }) \wedge(\text { last } \rightarrow \neg \text { wmark }) \wedge(\text { wmark } \rightarrow \text { wmark }[-1 \mid 1]) \wedge \\
(\neg \text { wmark } \rightarrow \neg \text { wmark }[+1 \mid 0]) \text { then } \text { wmark else } \neg \text { wmark }
\end{gathered}
$$

$\widetilde{\mathrm{E}}_{i}$ is obtained from $\mathrm{E}_{i}$ by replacing each stream subexpression $\tau[k \mid d]$ s.t. $k>0$ with: if $\bigvee_{j=1}^{j=k} \neg$ wmark $[j \mid 0]$ thend else $\tau[k \mid d]$

$\widetilde{\mathrm{E}}_{i}^{\prime}$ is obtained from $\mathrm{E}_{i}^{\prime}$ by replacing each stream subexpression $\tau[k \mid d]$ s.t. $k<0$ with:

$$
\text { if } \bigvee_{j=1}^{j=-k} \text { wmark }[-j \mid 1] \text { then d else } \tau[k \mid d]
$$

Fig. 3. Construction for concatenation

guessed decomposition of the input $\sigma_{X}$, whether $k+j$ is still a position inside $\sigma_{X, i}$.

Concatenation. The construction is given in Fig. 3. We assume w.l.o.g. that the BSRV $\varphi$ and $\varphi^{\prime}$ have no output variables in common. The BSRV recognizing $\mathcal{L}(\varphi) \cdot \mathcal{L}\left(\varphi^{\prime}\right)$ uses a new output variable: wmark. This variable is used for guessing a decomposition in the given input of the form $\sigma_{X} \cdot \sigma_{X}^{\prime}$ in such a way that $\sigma_{X} \in \mathcal{L}(\varphi)$ and $\sigma_{X}^{\prime} \in \mathcal{L}\left(\varphi^{\prime}\right)$. In particular, the output variable wmark assumes the value 1 along all and only the positions of $\sigma_{X}$ (the equation for wmark ensures that a Boolean stream for wmark is always in $\left.1^{+} 0^{+}\right)$. Moreover, the equations for the output variables of $\varphi$ are modified in order to allow to check for a positive offset $k>0$ of $\varphi$ and a position $j$ inside $\sigma_{X}$ in the guessed decomposition $\sigma_{X} \cdot \sigma_{X}^{\prime}$ of the input, whether $k+j$ is still a position inside $\sigma_{X}$. Analogously, the equations 




Fig. 4. Offline monitoring algorithm for well-defined BSRV

for the output variables of $\varphi^{\prime}$ are modified to allow checking for a negative offset $k<0$ of $\varphi^{\prime}$ and a position $j$ inside $\sigma_{X}^{\prime}$ in the guessed decomposition $\sigma_{X} \cdot \sigma_{X}^{\prime}$ of the input, whether $k+j$ is still a position inside $\sigma_{X}^{\prime}$.

\section{Offline Monitoring for Well-defined BSRV}

In this section, we propose an offline monitoring algorithm for well-defined BSRV based on Theorem 1. The algorithm runs in time linear in the length of the input trace (input streams) and singly exponential in the size of the specification. Additionally, we partially solve a question left open in [8] for the case of BSRV.

Let $\varphi$ be a BSRV over $X$ and $Y$, and $\mathcal{A}_{\varphi}=\left\langle Q, q_{0}, \delta, F\right\rangle$ be the NFA over $2^{X}$ accepting $\mathcal{L}(\varphi)$ of Theorem 1. Recall that $Q \backslash\left\{q_{0}\right\}$ is contained in $\left(A_{\perp}\right)^{b(\varphi)} \times$ $A \times\left(A_{\perp}\right)^{f(\varphi)}$, where $A=2^{X \cup Y}$ and $A_{\perp}:=A \cup\{\perp\}$, and an expanded valuation model of $\varphi$ is of the form $\pi=q_{0}, q_{1}, \ldots, q_{k}$, where $q_{i} \in Q \backslash\left\{q_{0}\right\}$ for all $1 \leq i \leq k$. Moreover, the valuation model of $\varphi$ encoded by $\pi$ is the sequence of the main values of the states $q_{i}$ visited by $\pi$. By Theorem 1 , the set of accepting runs of $\mathcal{A}_{\varphi}$ over an input $\sigma_{X}$ is the set of expanded valuation models of $\varphi$ encoding the valuation models of $\varphi$ associated with the input $\sigma_{X}$. Hence, the following holds.

Proposition 2. A BSRV $\varphi$ is well-defined if and only if the NFA $\mathcal{A}_{\varphi}$ is universal and unambiguous.

The offline monitoring algorithm for well-defined BSRV is given in Fig. 4, where we assume that the input trace $\sigma_{X}$ is available on a tape. The algorithm operates in two phases. In the first phase, a forward traversing of the input trace is performed, and the algorithm simulates the unique run over the input $\sigma_{X}$ of the deterministic finite state automaton (DFA) that would result from $\mathcal{A}_{\varphi}$ by the classical powerset construction. Let $\left\{q_{0}\right\}, \Lambda(1), \ldots, \Lambda\left(\left|\sigma_{X}\right|\right)$ be the run of this DFA over $\sigma_{X}$. Then, at each step $i$, the state $\Lambda(i)$ of the run resulting from reading the input symbol $\sigma_{X}(i)$ is stored in the $i$ th position of the tape. In the second phase, a backward traversing of the input trace is performed, and the algorithm outputs a stream valuation of $\varphi$. Since $\varphi$ is well-defined, by using 
Proposition 2, we easily deduce that the uniqueness conditions in the second phase of the algorithm are satisfied. Moreover, the sequence of states computed by the algorithm in the second phase is the unique accepting run $\pi$ of $\mathcal{A}_{\varphi}$ over $\sigma_{X}$. Therefore, the algorithm outputs the valuation model of $\varphi$ encoded by $\pi$, which is the unique valuation model of $\varphi$ associated with the input $\sigma_{X}$. Thus, since the size of the NFA $\mathcal{A}_{\varphi}$ is singly exponential in the size of $\varphi$, we obtain the following result. (for a detailed proof of Theorem 5, see Appendix B.1).

Theorem 5. One can construct an offline monitoring algorithm for well-defined BSRV running in time linear in the length of the input trace and singly exponential in the size of the specification. Additionally, the algorithm processes a position of the input trace exactly twice.

In [8], a syntactical condition for general SRV, called well-formedness, is introduced, which can be checked in polynomial time and implies well-definedness. Well-formedness ensures the absence of circular definitions by requiring that a dependency graph of the output variables have not zero-weight cycles. As illustrated in [8], for the restricted class of well-formed SRV, it is possible to construct an offline monitoring algorithm which runs in time linear in the length of the input trace and the size of the specification. Moreover, one can associate to a well-formed $\operatorname{SRV} \varphi$ a parameter $\operatorname{ad}(\varphi)$, called alternation depth [8], such that the monitoring algorithm processes each position of the input trace exactly $a d(\varphi)+1$ times. An important question left open in [8] is whether for a well-formed SRV $\varphi$, it is possible to construct a $\varphi$-equivalent SRV whose alternation depth is minimal. Here, we settle partially this question for the class of BSRV. By using the same ideas for constructing the algorithm of Fig. 4, we show that for the class of BSRV, the semantic notion of well-definedness coincides with the syntactical notion of well-formedness (modulo BSRV-equivalence), and the hierarchy of well-formed BSRV induced by the alternation depth collapses to the level 1 . In particular, we establish the following result (the full proof can be found in Appendix B.2.

Theorem 6. Given a well-defined BSRV $\varphi$, one can build in doubly exponential time a $\varphi$-equivalent BSRV which is well-formed and has alternation depth 1.

\section{Decision Problems}

We investigate complexity issues for some relevant decision problems on BSRV. In particular, we establish that while checking well-definedness is in EXPTIME, checking for a given BSRV $\varphi$ and a given subset $Z$ of output variables, whether $\varphi$ is well-defined with respect to $Z$ (generalized well-definedness problem) is instead EXPSPACE-complete. Our results can be summarized as follows.

Theorem 7. For BSRV:

1. The under-definedness problem is PSPACE-complete, the well-definedness problem is in EXPTIME and at least PSPACE-hard, while the over-definedness problem and the generalized well-definedness problem are both EXPSPACEcomplete. 
2. Checking semantic equivalence is EXPSPACE-complete.

3. When interpreted as language recognizers, language emptiness is PSPACEcomplete, while language universality, language inclusion, and language equivalence are EXPSPACE-complete.

Here, we illustrate the upper bounds of Theorem 7(1). Detailed proofs of all results can be found in Appendix 5. We need a preliminary result (Proposition 3). For an NFA $\mathcal{A}=\left\langle Q, q_{0}, \delta, F\right\rangle$, a state projection of $\mathcal{A}$ is a mapping $\Upsilon: Q \rightarrow P$ for some finite set $P$ such that for all $q \in Q, \Upsilon(q)$ is computable in logarithmic space (in the size of $Q$ ). The mapping $\Upsilon$ can be extended to sequences of states in the obvious way. We say that the NFA $\mathcal{A}$ is unambiguous with respect to $\Upsilon$ if for all $w \in \mathcal{L}(\mathcal{A})$ and accepting runs $\pi$ and $\pi^{\prime}$ of $\mathcal{A}$ over $w$, their projections $\Upsilon(\pi)$ and $\Upsilon\left(\pi^{\prime}\right)$ coincide.

Proposition 3. Given an NFA $\mathcal{A}$ and a state projection $\Upsilon$ of $\mathcal{A}$, checking whether $\mathcal{A}$ is not unambiguous with respect to $\Upsilon$ can be done in NLOGSPACE.

Upper Bounds of Theorem 7(1). Let $\varphi$ be a BSRV over $X$ and $Y$, and $\mathcal{A}_{\varphi}$ be the NFA of Theorem 1 accepting $\mathcal{L}(\varphi)$ and whose size is singly exponential in the size of $\varphi$.

Under-definedness: by Theorem 1 and Lemma $1, \varphi$ is under-defined iff $\mathcal{A}_{\varphi}$ is not unambiguous. Thus, since $\mathcal{A}_{\varphi}$ can be constructed on the fly and PSPACE $=$ NPSPACE, by Proposition 3 (with $\Upsilon$ being the identity map), it follows that the under-definedness problem is in PSPACE.

Over-definedness: since $\mathcal{A}_{\varphi}$ accepts $\mathcal{L}(\varphi), \varphi$ is over-defined iff $\mathcal{A}_{\varphi}$ is not universal. Thus, since checking universality for NFA is a well-known PSPACE-complete problem [20], membership in EXPSPACE for checking over-definedness follows.

Well-definedness: it is well-known that checking universality of unambiguous NFA can be done in polynomial time [25]. By Proposition 2, $\varphi$ is well-defined iff $\mathcal{A}_{\varphi}$ is universal and unambiguous. Thus, since checking that $\mathcal{A}_{\varphi}$ is unambiguous can be done in PSPACE (in the size of $\varphi$ ), membership in EXPTIME for checking well-definedness follows.

Generalized Well-definedness: let $Z \subseteq Y$. Recall that the set of non-initial states of $\mathcal{A}_{\varphi}$ is contained in $\left(A_{\perp}\right)^{b(\varphi)} \times A \times\left(A_{\perp}\right)^{f(\varphi)}$, where $A=2^{X \cup Y}$ and $A_{\perp}:=A \cup$ $\left\{\perp\right.$. Let $\Upsilon_{Z}$ be the state projection of $\mathcal{A}_{\varphi}$ assigning to the initial state $q_{0}$ of $\mathcal{A}_{\varphi}$ $q_{0}$ itself, and assigning to each non-initial state $\left(a_{-b(\varphi)}, \ldots, a_{-1}, a_{0}, a_{1}, \ldots, a_{f(\varphi)}\right)$ of $\mathcal{A}_{\varphi}$ the tuple $\left(d_{-b(\varphi)}, \ldots, d_{-1}, d_{0}, d_{1}, \ldots, d_{f(\varphi)}\right)$, where for all $b(\varphi) \leq i \leq f(\varphi)$, $d_{i}=a_{i}$ if $a_{i}=\perp$, and $d_{i}=a_{i} \cap Z$ otherwise. Now, let $\sigma$ and $\sigma^{\prime}$ be two valuation models of $\varphi$ associated with an input $\sigma_{X}$, and $\pi$ and $\pi^{\prime}$ be the expanded valuation models encoding $\sigma$ and $\sigma^{\prime}$, respectively. By construction, it follows that $\Upsilon_{Z}(\pi)=\Upsilon_{Z}\left(\pi^{\prime}\right)$ iff the restrictions of $\sigma$ and $\sigma^{\prime}$ to $Z$ coincide. By Theorem 1 , we obtain that $\varphi$ is well-defined with respect to $Z$ iff $\mathcal{A}_{\varphi}$ is unambiguous with respect to $\Upsilon_{Z}$ and $\mathcal{A}_{\varphi}$ is universal. Thus, since checking universality for NFA is PSPACE-complete, by Proposition 3, membership in EXPSPACE for checking generalized well-definedness follows. 


\section{Conclusion}

In this paper, we have studied some theoretical problems for the class of Boolean SRV. We have also presented an offline monitoring algorithm for well-defined BSRV that only requires two passes over the dumped trace. An open question is the exact complexity of checking well-definedness for BSRV: it lies somewhere between PSPACE and EXPTIME. Future work includes the theoretical investigation and the development of monitoring algorithms for SRV over richer data types, such as counters and stacks. In particular, the emerging field of symbolic automata and transducers [26] — that extend the classical notions from discrete alphabets to theories handled by solvers - seems very promising to study in the context of SRV, which in turn can extend automata from states and transitions to stream dependencies. The combination of these two extensions has the potential to provide a rich but tractable foundation for the runtime verification of values from rich types. Additionally, we are studying the extension to the monitoring of visibly pushdown systems, where SRV is extended to deal with traces containing calls and returns.

Finally, we plan to study the monitorability of well-definedness of specifications. If one cannot determine well-definedness statically, a plausible alternative would be to use a monitor that assumes well-definednees in tandem with a monitor that detects non-well-definedness (and hence, the incorrectness of the first monitor).

\section{References}

1. H. Barringer, A. Goldberg, K. Havelund, and K. Sen. Rule-based runtime verification. In Proc. of VMCAI'04, LNCS 2937, pages 44-57. Springer, 2004.

2. D. Basin, M. Harvan, F. Klaedtke, and E. Zălinescu. MONPOLY: Monitoring usage-control policies. In Proc. of RV'12, LNCS 7687. Springer, 2012.

3. D. Basin, F. Klaedtke, and S. Müller. Policy monitoring in first-order temporal logic. In Proc. of $C A V^{\prime} 10$, LNCS 6174, pages 1-18. Springer, 2010.

4. A. Bauer, R. Gore, and A. Tiu. A first-order policy language for history-based transaction monitoring. In Proc. of ICTAC'09, LNCS 5684, pages 96-111. Springer, 2009.

5. A. Bauer, M. Leucker, and C. Schallhart. Runtime verification for LTL and TLTL. ACM Transactions on Software Engineering and Methodology, 20(4):14, 2011.

6. G. Berry. Proof, language, and interaction: essays in honour of Robin Milner, chapter The foundations of Esterel, pages 425-454. MIT Press, 2000.

7. P. Caspi and M. Pouzet. Synchronous Kahn Networks. In Proc. of ICFP'96, pages 226-238. ACM Press, 1996.

8. B. D'Angelo, S. Sankaranarayanan, C. Sánchez, W. Robinson, B. Finkbeiner, H. B. Sipma, S. Mehrotra, and Z. Manna. LOLA: Runtime monitoring of synchronous systems. In Proc. of TIME'05, pages 166-174. IEEE CS Press, 2005.

9. C. Eisner, D. Fisman, J. Havlicek, Y. Lustig, A. McIsaac, and D. V. Campenhout. Reasoning with temporal logic on truncated paths. In Proc. of $C A V^{\prime} 03$, volume 2725 of $L N C S$ 2725, pages 27-39. Springer, 2003.

10. B. Finkbeiner, S. Sankaranarayanan, and H. B. Sipma. Collecting statistics over runtime executions. ENTCS, 70(4):36-54, 2002. 
11. T. Gautier, P. Le Guernic, and L. Besnard. SIGNAL: A declarative language for synchronous programming of real-time systems. In Proc. of FPCA'87, LNCS 274, pages 257-277. Springer, 1987.

12. A. E. Goodloe and L. Pike. Monitoring distributed real-time systems: A survey and future directions. Technical report, NASA Langley Research Center, 2010.

13. N. Halbwachs, P. Caspi, D. Pilaud, and J. Plaice. Lustre: a declarative language for programming synchronous systems. In Proc. of POPL'87, pages 178-188. ACM Press, 1987.

14. D. Harel. The spirit of Computing. Addison-Wesley, 2nd edition, 1992.

15. K. Havelund and A. Goldberg. Verify your runs. In Proc. of VSTTE'05, LNCS 4171, pages 374-383. Springer, 2005.

16. K. Havelund and G. Roşu. Synthesizing monitors for safety properties. In Proc. of TACAS'02, LNCS 2280, pages 342-356. Springer, 2002.

17. F. Laroussinie, N. Markey, and P. Schnoebelen. Temporal logic with forgettable past. In Proc. of LICS'02, pages 383-392. IEEE CS Press, 2002.

18. M. Leucker and C. Schallhart. A brief account of runtime verification. The Journal of Logic and Algebraic Programming, 78(5):293-303, 2009.

19. Z. Manna and A. Pnueli. Temporal Verification of Reactive Systems: Safety. Springer, New York, 1995.

20. A. R. Meyer and L. J. Stockmeyer. The equivalence problem for regular expressions with squaring requires exponential space. In Proc. of FOCS'r2, pages 125-129. IEEE CS Press, 1972.

21. L. Pike, A. Goodloe, R. Morisset, and S. Niller. Copilot: A hard real-time runtime monitor. In Proc. of RV'10, LNCS 6418. Springer, 2010.

22. A. Pnueli and A. Zaks. PSL model checking and run-time verification via testers. In Proc. of FM'06, LNCS 4085, pages 573-586. Springer, 2006.

23. G. Roşu and K. Havelund. Rewriting-based techniques for runtime verification. Autom. Softw. Eng., 12(2):151-197, 2005.

24. K. Sen and G. Roşu. Generating optimal monitors for extended regular expressions. ENTCS, 89(2):226-245, 2003.

25. R. E. Stearns and H. B. Hunt. On the equivalence and containment problems for unambiguous regular expressions, regular grammars and finite automata. SIAM J. Comput., 14(3):598-611, 1985.

26. M. Veanes, P. Hooimeijer, B. Livshits, D. Molnar, and N. Bjrner. Symbolic finite state transducers: algorithms and applications. In Proc. of POPL'12, pages 137150. ACM, 2012. 


\section{Appendix}

\section{A Proofs from Section 3}

\section{A.1 Full proof of Theorem 4}

Theorem 4. BSRV are effectively closed under the following language operations: intersection, union, reversal, positive Kleene closure, and concatenation. Additionally, the constructions for these operations can be done in linear time.

Proof. Intersection. Let $\varphi$ be a BSRV over $X$ and $Y$ and $\varphi$ be a BSRV over $X$ and $Y^{\prime}$. Without loss of generality, we assume that $Y \cap Y^{\prime}=\emptyset$. Then, the BSRV $\varphi^{\prime \prime}$ recognizing $\mathcal{L}(\varphi) \cap \mathcal{L}\left(\varphi^{\prime}\right)$ is simply the joint set of the equations of $\varphi$ and $\varphi^{\prime}$. Correctness of the construction immediately follows.

Union. Let $\varphi=\left\{y_{1}=\mathrm{E}_{1}, \ldots, y_{k}=\mathrm{E}_{k}\right\}$ be a BSRV over $X$ and $Y=\left\{y_{1}, \ldots, y_{k}\right\}$, and $\varphi^{\prime}=\left\{y_{1}^{\prime}=\mathrm{E}_{1}^{\prime}, \ldots, y_{h}^{\prime}=\mathrm{E}_{h}^{\prime}\right\}$ be a BSRV over $X$ and $Y^{\prime}=\left\{y_{1}^{\prime}, \ldots, y_{h}^{\prime}\right\}$. We construct in linear time a BSRV $\varphi^{\prime \prime}$ with set of input variables $X$ and recognizing $\mathcal{L}(\varphi) \cup \mathcal{L}\left(\varphi^{\prime}\right)$ as follows. The set of output variables of $\varphi^{\prime \prime}$ is given by $Y \cup Y^{\prime} \cup$ \{check, main\}, where check and main are two new output variables. Intuitively, check is a uniform output variable used to guess whether the input $\sigma_{X}$ has to be considered an input for $\varphi$ or $\varphi^{\prime}$. The equation for check is given by

$$
\text { check }=\text { if } \neg \text { last } \rightarrow(\text { check } \leftrightarrow \text { check }[+1 \mid 1]) \text { then } \text { check else } \neg \text { check }
$$

Note that the boolean streams for check satisfying the above equation range over all uniform boolean streams. For each $y \in Y \cup Y^{\prime}$, the equation of $\varphi^{\prime \prime}$ associated to $y$ is simply $y=y$. Finally, the equation for the new output variable main is defined as follows

$$
\text { main }=\text { if }\left(\left(\text { check } \rightarrow \bigwedge_{i=1}^{i=k} y_{i} \leftrightarrow \mathrm{E}_{i}\right) \wedge\left(\neg \text { check } \rightarrow \bigwedge_{i=1}^{i=h} y_{i}^{\prime} \leftrightarrow \mathrm{E}^{\prime}{ }_{i}\right)\right) \text { then main }
$$

Now, we show that the construction is correct, i.e. $\mathcal{L}\left(\varphi^{\prime \prime}\right)=\mathcal{L}(\varphi) \cup \mathcal{L}\left(\varphi^{\prime}\right)$. For the inclusion $\mathcal{L}\left(\varphi^{\prime \prime}\right) \subseteq \mathcal{L}(\varphi) \cup \mathcal{L}\left(\varphi^{\prime}\right)$, let $\sigma_{X} \in \mathcal{L}\left(\varphi^{\prime \prime}\right)$. Hence, there is a valuation model $\sigma^{\prime \prime}$ of $\varphi^{\prime \prime}$ associated with the input $\sigma_{X}$. By construction, $\sigma^{\prime \prime}$ (check) $\in$ $0^{+} \cup 1^{+}$. Assume that $\sigma^{\prime \prime}$ (check) $\in 1^{+}$(the other case being similar). Then, by definition of the equation for main, it holds that for each $1 \leq i \leq k, \llbracket y_{i}, \sigma^{\prime \prime} \rrbracket=$ $\llbracket \mathrm{E}_{i}, \sigma^{\prime \prime} \rrbracket$. Thus, the restriction of $\sigma^{\prime \prime}$ to $X \cup Y$ is a valuation model of $\varphi$. Hence, $\sigma_{X} \in \mathcal{L}(\varphi)$ and the result follows. For the converse inclusion $\mathcal{L}(\varphi) \cup \mathcal{L}\left(\varphi^{\prime}\right) \subseteq$ $\mathcal{L}\left(\varphi^{\prime \prime}\right)$, let $\sigma_{X} \in \mathcal{L}(\varphi) \cup \mathcal{L}\left(\varphi^{\prime}\right)$. Assume that $\sigma_{X} \in \mathcal{L}\left(\varphi^{\prime}\right)$ (the other case being similar). Hence, there is a valuation model $\sigma^{\prime}$ of $\varphi^{\prime}$ associated with the input $\sigma_{X}$. Let $\sigma^{\prime \prime}$ be the stream valuation of $\varphi^{\prime \prime}$ associated with the input $\sigma_{X}$ defined as follows: for all variables $z$ of $\varphi^{\prime \prime}, \sigma^{\prime \prime}(z)=\sigma^{\prime}(z)$ if $z \in X \cup Y^{\prime}$, and $\sigma^{\prime \prime}(z) \in 0^{+}$ 
otherwise. By construction, it easily follows that $\sigma^{\prime \prime}$ is a valuation model of $\varphi^{\prime \prime}$. Hence, membership of $\sigma_{X}$ in $\mathcal{L}\left(\varphi^{\prime \prime}\right)$ directly follows.

Language Reversal. First, we need additional notation. For a finite word $w$ and a position $1 \leq i \leq|w|$, we denote by $w^{R}$ the reverse of $w$, and by $R(i)$ the position of $w$ given by $|w|-i+1$. Note that the suffix of $w^{R}$ from position $R(i)$ is the reverse of the prefix of $w$ leading to position $i$. Moreover, for a stream expression $\mathrm{E}$, we denote by $\mathrm{E}^{R}$ the stream expression obtained from E by replacing each subexpression $\tau[k \mid d]$ (resp. $\tau[-k \mid d]$ ) with $k>0$ with the subexpression $\tau[-k \mid d]$ (resp., $\tau[k \mid d]$ ). By structural induction, one can easily show that for a stream valuation $\sigma$ over the variables of $\mathrm{E}$ and for all $1 \leq i \leq|\sigma|$, it holds that $\llbracket \mathrm{E}, \sigma \rrbracket(i)=\llbracket \mathrm{E}^{R}, \sigma^{R} \rrbracket(R(i))$. Now, let $\varphi$ be a BSRV over $X$ and $Y$. The BSRV $\varphi^{R}$ accepting the reversal of $\mathcal{L}(\varphi)$ is obtained from $\varphi$ by converting each equation $y=\mathrm{E}$ of $\varphi$ in the equation $y=\mathrm{E}^{R}$. Let $\sigma$ be a stream valuation over $X \cup Y$. Since $\llbracket \mathrm{E}, \sigma \rrbracket(i)=\llbracket \mathrm{E}^{R}, \sigma^{R} \rrbracket(R(i))$ for all stream expressions $\mathrm{E}$ over $X \cup Y$ and position $i$ of $\sigma$, by construction, it follows that $\sigma$ is a valuation model of $\varphi$ if and only if $\sigma^{R}$ is a valuation model of $\varphi^{R}$. Hence, the equality $\mathcal{L}\left(\varphi^{R}\right)=[\mathcal{L}(\varphi)]^{R}$ directly follows.

Positive Kleene closure. Let $\varphi$ be a BSRV over $X$ and $Y$. We construct in linear time a BSRV $\varphi^{\prime \prime}$ with set of input variables $X$ and recognizing $[\mathcal{L}(\varphi)]^{+}$as follows. The set $Y^{\prime \prime}$ of output variables of $\varphi^{\prime \prime}$ is obtained from $Y$ by adding two new output variables, wbegin and wend. Intuitively, these new output variables are used for guessing a decomposition in the input $\sigma_{X}$ of the form $\sigma_{X}=\sigma_{X, 1} \cdot \ldots$. $\sigma_{X, N}$ for some $N \geq 1$ in such a way that each component $\sigma_{X, i}$ is in $\mathcal{L}(\varphi)$. In particular, the output variable wbegin (resp., wend) is used to mark the first (resp., the last) positions of the components $\sigma_{X, i}$. Formally, the equations for the output variables wbegin and wend are defined as follows.

wbegin $=$ if $($ first $\rightarrow$ wbegin $) \wedge($ wbegin $\rightarrow$ wend $[-1 \mid 1])$ then wbegin else $\neg$ wbegin wend $=$ if (last $\rightarrow$ wend $) \wedge$ (wend $\rightarrow$ wbegin $[+1 \mid 1]$ ) then wend else $\neg$ wend

Note that for a stream valuation $\sigma$ satisfying the above two equations, $\sigma$ can be decomposed in the form $\sigma=\sigma_{1} \cdot \ldots \cdot \sigma_{N}$ for some $N \geq 1$ such that $\sigma_{i}$ (wbegin) $\in$ $10^{*}$ and $\sigma_{i}$ (wend) $\in 0^{*} 1$ for all $1 \leq i \leq N$. Finally, for each output variable $y \in Y$, the equation for $y$ of the specification $\varphi^{\prime \prime}$ is obtained from the equation $y=\mathrm{E}$ of $\varphi$ by replacing each stream subexpression $\tau[k \mid d]$ occurring in $\mathrm{E}$, with the stream expression $\mathrm{E}_{\tau, k, d}$ defined as follows:

$$
\mathrm{E}_{\tau, k, d}= \begin{cases}\text { if } \bigvee_{\substack{j=1 \\ j=-k}} \text { wbegin }[j \mid 1] \text { then d else } \tau[k \mid d] \quad \text { if } k>0 \\ \text { if } \bigvee_{j=1} \text { wend }[-j \mid 1] \text { then d else } \tau[k \mid d] \text { if } k<0\end{cases}
$$

Now, we show that the construction is correct, i.e. $\mathcal{L}\left(\varphi^{\prime \prime}\right)=[\mathcal{L}(\varphi)]^{+}$. For the inclusion $\mathcal{L}\left(\varphi^{\prime \prime}\right) \subseteq[\mathcal{L}(\varphi)]^{+}$, let $\sigma_{X} \in \mathcal{L}\left(\varphi^{\prime \prime}\right)$. Hence, there is a valuation model $\sigma$ 
of $\varphi^{\prime \prime}$ associated with the input $\sigma_{X}$. By construction, there is $N \geq 1$ such that $\sigma$ can be written in the form $\sigma=\sigma_{1} \cdot \ldots \cdot \sigma_{N}$ and for all $1 \leq \ell \leq N$, the boolean stream $\sigma_{\ell}$ (wbegin) is in $10^{*}$ and the boolean stream $\sigma_{\ell}$ (wend) is in $0^{*} 1$. We show that the restriction $\left(\sigma_{\ell}\right)_{X \cup Y}$ of $\sigma_{\ell}$ to the set of variables $X \cup Y$ is a valuation model of $\varphi$. Hence, membership of $\sigma_{X}$ in $[\mathcal{L}(\varphi)]^{+}$follows. Fix $1 \leq \ell \leq N$. For all positions $1 \leq i \leq\left|\sigma_{\ell}\right|$ along the stream valuation $\sigma_{\ell}$, we denote by $p(i)$ the corresponding position along $\sigma$. In order to prove that $\sigma_{\ell}$ is a valuation model of $\varphi$, by hypothesis, it suffices to show that for each equation $y=\mathrm{E}$ of $\varphi$ and for each position $1 \leq i \leq\left|\sigma_{\ell}\right|$, the following holds, where $y=\mathrm{E}^{\prime \prime}$ is the equation of $\varphi^{\prime \prime}$ associated to the variable $y$ : $\mathbb{E},\left(\sigma_{\ell}\right)_{X \cup Y} \rrbracket(i)=\llbracket \mathrm{E}^{\prime \prime}, \sigma \rrbracket(p(i))$. Evidently, by construction, we just need to prove that for each subexpression $\tau[k \mid d]$ of $\varphi$, $\llbracket \tau[k \mid d],\left(\sigma_{\ell}\right)_{X \cup Y} \rrbracket(i)=\llbracket \mathrm{E}_{\tau, k, d}, \sigma \rrbracket(p(i))$. There are two cases:

$-k>0$ : first, assume that $i+k \leq\left|\sigma_{\ell}\right|$. Since $\sigma_{\ell}$ (wbegin) is in $10^{*}$, we obtain that for all $1 \leq j \leq k, \sigma$ (wbegin $)(p(i)+j)=0$. Hence, by definition of $\mathrm{E}_{\tau, k, d}$, it follows that $\llbracket \overline{\mathrm{E}}_{\tau, k, d}, \sigma \rrbracket(p(i))=\llbracket \tau[k \mid d], \sigma \rrbracket(p(i))=\llbracket \tau[k \mid d],\left(\sigma_{\ell}\right)_{X \cup Y} \rrbracket(i)$, and the result follows in this case. Now, assume that $i+k>\left|\sigma_{\ell}\right|$. Hence, $\llbracket \tau[k \mid d],\left(\sigma_{\ell}\right)_{X \cup Y} \rrbracket(i)=d$. Then, either $p(i)+k>|\sigma|$, or there is $1 \leq j \leq k$ such that $\sigma$ (wbegin $)(p(i)+j)=1$. By definition of $\mathrm{E}_{\tau, k, d}$, it follows that $\llbracket \mathrm{E}_{\tau, k, d}, \sigma \rrbracket(p(i))=d$, and the result follows in this case as well.

$-k<0$ : first, assume that $i-k \geq 1$. Since $\sigma_{\ell}$ (wend) is in $0^{*} 1$, we obtain that for all $1 \leq j \leq-k, \sigma$ (wend) $(p(i)-j)=0$. Hence, by definition of $\mathrm{E}_{\tau, k, d}$, it follows that $\llbracket \mathrm{E}_{\tau, k, d}, \sigma \rrbracket(p(i))=\llbracket \tau[k \mid d], \sigma \rrbracket(p(i))=\llbracket \tau[k \mid d],\left(\sigma_{\ell}\right)_{X \cup Y} \rrbracket(i)$, and the result follows in this case. Now, assume that $i-k<1$. Hence, $\llbracket \tau[k \mid d],\left(\sigma_{\ell}\right)_{X \cup Y} \rrbracket(i)=$ $d$. Then, either $p(i)-k<1$, or there is $1 \leq j \leq-k$ such that $\sigma$ (wend) $(p(i)-$ $j)=1$. By definition of $\mathrm{E}_{\tau, k, d}$, it follows that $\llbracket \mathrm{E}_{\tau, k, d}, \sigma \rrbracket(p(i))=d$, and the result follows in this case as well.

For the converse inclusion $[\mathcal{L}(\varphi)]^{+} \subseteq \mathcal{L}\left(\varphi^{\prime \prime}\right)$, let $\sigma_{X} \in[\mathcal{L}(\varphi)]^{+}$. Hence, there is a stream valuation $\sigma$ of $\varphi$ associated with the input $\sigma_{X}$ such that $\sigma$ is of the form $\sigma=\sigma_{1} \cdots \sigma_{N}$ for some $N \geq 1$, and $\sigma_{\ell}$ is a valuation model of $\varphi$ for all $1 \leq \ell \leq N$. Let $\sigma^{\prime \prime}=\sigma_{1}^{\prime \prime} \cdots \sigma_{N}^{\prime \prime}$ be the extension of $\sigma$ over $X \cup Y^{\prime \prime}$, where the boolean streams $\sigma^{\prime \prime}$ (wbegin) and $\sigma^{\prime \prime}$ (wend) are defined as follows: for all $1 \leq \ell \leq N, \sigma_{\ell}^{\prime \prime}$ (wbegin) is in $10^{*}$ and the boolean stream $\sigma_{\ell}^{\prime \prime}$ (wend) is in $0^{*} 1$. We show that $\sigma^{\prime \prime}$ is a valuation model of $\varphi^{\prime \prime}$, hence, membership of $\sigma_{X}$ in $\mathcal{L}\left(\varphi^{\prime \prime}\right)$ follows. By construction, the equations for the output variables wbegin and wend are satisfied with respect to the stream valuation $\sigma^{\prime \prime}$. Now, let $\mathrm{u} s$ consider an equation $y=\mathrm{E}^{\prime \prime}$ of $\varphi^{\prime \prime}$ associated with an output variable $y \in Y$. By construction and the hypothesis, in order to show that $\llbracket y, \sigma^{\prime \prime} \rrbracket=\llbracket \mathrm{E}, \sigma^{\prime \prime} \rrbracket$, it suffices to prove that for all $1 \leq \ell \leq N, 1 \leq i \leq\left|\sigma_{\ell}\right|$, and subsexpression $\tau[k \mid d]$ of $\varphi, \llbracket \tau[k \mid d], \sigma_{\ell} \rrbracket(i)=\llbracket \mathrm{E}_{\tau, k, d}, \bar{\sigma}^{\prime \prime} \rrbracket(p(i))$, where for $1 \leq i \leq\left|\sigma_{\ell}\right|, p(i)$ denotes the corresponding position along $\sigma$. This can be shown as for the proof of the inclusion $\mathcal{L}\left(\varphi^{\prime \prime}\right) \subseteq[\mathcal{L}(\varphi)]^{+}$, and we omit the details here.

Concatenation. Let $\varphi$ be a BSRV over $X$ and $Y$ and $\varphi$ be a BSRV over $X$ and $Y^{\prime}$. Without loss of generality, we assume that $Y \cap Y^{\prime}=\emptyset$. We construct in linear time a BSRV $\varphi^{\prime \prime}$ with set of input variables $X$ and recognizing $\mathcal{L}(\varphi) \cup \mathcal{L}\left(\varphi^{\prime}\right)$ as 
follows. The set $Y^{\prime \prime}$ of output variables of $\varphi^{\prime \prime}$ is given by $Y^{\prime \prime}=Y \cup Y^{\prime} \cup\{$ wmark $\}$, where wmark is a new output variable. Intuitively, wmark is used for guessing a decomposition in the input $\sigma_{X}^{\prime \prime}$ of the form $\sigma_{X}^{\prime \prime}=\sigma_{X} \cdot \sigma_{X}^{\prime}$ in such a way that $\sigma_{X} \in \mathcal{L}(\varphi)$ and $\sigma_{X}^{\prime} \in \mathcal{L}\left(\varphi^{\prime}\right)$. In particular, the output variable wmark assume the value 1 along all and only the positions of $\sigma_{X}$. Formally, the equation for the output variable wmark is defined as follows.

$$
\begin{aligned}
\text { wmark }=\text { if } & (\text { first } \rightarrow \text { wmark }) \wedge(\text { last } \rightarrow \neg \text { wmark }) \wedge(\text { wmark } \rightarrow \text { wmark }[-1 \mid 1]) \wedge \\
& (\neg \text { wmark } \rightarrow \neg \text { wmark }[+1 \mid 0]) \text { then } \text { wmark else } \neg \text { wmark }
\end{aligned}
$$

Note that a boolean stream for wmark is always in $1^{+} 0^{+}$. The equations of $\varphi^{\prime \prime}$ for the remaining output variables are defined as follows. For each stream expression $\tau[k, d]$, let $\mathrm{E}_{\tau, k, d}$ be the stream expression defined as follows:

$$
\mathrm{E}_{\tau, k, d}= \begin{cases}\text { if } \bigvee_{\substack{j=1 \\ j=-k}}^{\substack{j=k \\ \text { if }}} \bigvee_{j=1} \text { wmark }[j \mid 0] \text { then d else } \tau[k \mid d] & \text { if } k>0\end{cases}
$$

First, we consider the output variables $y \in Y$. Let $y=\mathrm{E}$ be the equation of $\varphi$ associated with $y$. Then, the equation for $y$ of the specification $\varphi^{\prime \prime}$ is given by $y=$ if wmark then $\mathrm{E}^{\prime \prime}$ else $y$, where $\mathrm{E}^{\prime \prime}$ is obtained from $\mathrm{E}$ by replacing each stream subexpression $\tau[k \mid d]$ occurring in $\mathrm{E}$ such that $k>0$ with the stream expression $\mathrm{E}_{\tau, k, d}$. Now, we consider the output variables $y \in Y^{\prime}$. Let $y=\mathrm{E}$ be the equation of $\varphi^{\prime}$ associated with $y$. Then, the equation for $y$ of the specification $\varphi^{\prime \prime}$ is given by $y=$ if $\neg$ wmark then $\mathrm{E}^{\prime \prime}$ else $y$, where $\mathrm{E}^{\prime \prime}$ is obtained from $\mathrm{E}$ by replacing each stream subexpression $\tau[k \mid d]$ occurring in $\mathbf{E}$ such that $k<0$ with the stream expression $\mathrm{E}_{\tau, k, d}$.

Now, we show that the construction is correct, i.e. $\mathcal{L}\left(\varphi^{\prime \prime}\right)=\mathcal{L}(\varphi) \cdot \mathcal{L}\left(\varphi^{\prime}\right)$. For the inclusion $\mathcal{L}\left(\varphi^{\prime \prime}\right) \subseteq \mathcal{L}(\varphi) \cdot \mathcal{L}\left(\varphi^{\prime}\right)$, let $\sigma_{X} \in \mathcal{L}\left(\varphi^{\prime \prime}\right)$. Hence, there is a valuation model $\sigma^{\prime \prime}$ of $\varphi^{\prime \prime}$ associated with the input $\sigma_{X}$. By construction, $\sigma^{\prime \prime}$ can be written in the form $\sigma^{\prime \prime}=\sigma \cdot \sigma^{\prime}$ such that $\sigma$ (wmark) is in $1^{+}$and $\sigma^{\prime}$ (wmark) is in $0^{+}$. We show that the restriction $(\sigma)_{X \cup Y}$ of $\sigma$ to the set of variables $X \cup Y$ is a valuation model of $\varphi$, and the restriction $(\sigma)_{X \cup Y^{\prime}}$ of $\sigma^{\prime}$ to the set of variables $X \cup Y^{\prime}$ is a valuation model of $\varphi^{\prime}$. Hence, membership of $\sigma_{X}$ in $\mathcal{L}(\varphi) \cdot \mathcal{L}\left(\varphi^{\prime}\right)$ follows. We consider the stream valuation $(\sigma)_{X \cup Y}$ (the proof for $(\sigma)_{X \cup Y^{\prime}}$ is similar). In order to prove that $(\sigma)_{X \cup Y}$ is a valuation model of $\varphi$, by hypothesis and construction, it suffices to show that for each equation $y=\mathrm{E}$ of $\varphi$ and for each position $1 \leq i \leq|\sigma|$, the following holds, where $y=$ if wmark then $\mathrm{E}^{\prime \prime}$ else $y$ is the equation of $\varphi^{\prime \prime}$ associated to the variable $y$ : $\llbracket \mathrm{E},(\sigma)_{X \cup Y} \rrbracket(i)=\llbracket \mathrm{E}^{\prime \prime}, \sigma^{\prime \prime} \rrbracket(i)$. Evidently, by construction, we just need to prove that for each subexpression $\tau[k \mid d]$ of $\varphi$ such that $k>0, \llbracket \tau[k \mid d],(\sigma)_{X \cup Y} \rrbracket(i)=\llbracket \mathrm{E}_{\tau, k, d}, \sigma^{\prime \prime} \rrbracket(i)$. This can be done similarly to the proof for the positive Kleene closure, and we omit the details here.

For the converse inclusion $\mathcal{L}(\varphi) \cdot \mathcal{L}\left(\varphi^{\prime}\right) \subseteq \mathcal{L}\left(\varphi^{\prime \prime}\right)$, let $\sigma_{X}^{\prime \prime} \in \mathcal{L}(\varphi) \cdot \mathcal{L}\left(\varphi^{\prime}\right)$. Hence, $\sigma_{X}^{\prime \prime}=\sigma_{X} \cdot \sigma_{X}^{\prime}$ and there are a valuation model $\sigma_{m}$ of $\varphi$ associated with the input 
$\sigma_{X}$ and a valuation model $\sigma_{m}^{\prime}$ of $\varphi^{\prime}$ associated with the input $\sigma_{X}^{\prime}$. Let $\sigma^{\prime \prime}$ be the stream valuation over $X \cup Y \cup Y^{\prime} \cup\{$ wmark $\}$ associated with the input $\sigma_{X}^{\prime \prime}$ defined as follows: $\sigma^{\prime \prime}=\sigma \cdot \sigma^{\prime}$ with $|\sigma|=\left|\sigma_{X}\right|$, where (i) $\sigma(z)=\sigma_{m}(z)$ if $z \in X \cup Y$, and $\sigma(z)=1$ otherwise, and (ii) $\sigma^{\prime}(z)=\sigma_{m}^{\prime}(z)$ if $z \in X \cup Y^{\prime}$, and $\sigma^{\prime}(z)=0$ otherwise. By construction, one can easily show that $\sigma^{\prime \prime}$ is a valuation model of $\varphi^{\prime \prime}$. Hence, membership of $\sigma_{X}^{\prime \prime}$ in $\mathcal{L}\left(\varphi^{\prime \prime}\right)$ follows.

\section{B Proofs from Section 4}

\section{B.1 Proof of Theorem 5}

Theorem 5. One can construct an offline monitoring algorithm for well-defined BSRV running in time linear in the length of the input trace and singly exponential in the size of the specification. Additionally, the algorithm processes a position of the input trace exactly twice.

Proof. The offline monitoring algorithm for well-defined BSRV is given in Figure 4 , where we assume that the input trace $\sigma_{X}$ is available on a tape. The algorithm operates in two phases. In the first phase, a forward traversing of the input trace is performed, and the algorithm simulates the unique run over the input $\sigma_{X}$ of the deterministic finite state automaton (DFA) resulting from $\mathcal{A}_{\varphi}$ by the classical powerset construction. Let $\left\{q_{0}\right\}, \Lambda(1), \ldots, \Lambda\left(\left|\sigma_{X}\right|\right)$ be the run of this DFA over $\sigma_{X}$. Then, at each step $i$, the state $\Lambda(i)$ of the run resulting from reading the input symbol $\sigma_{X}(i)$ is stored in the $i$ th position of the tape. In the second phase, a backward traversing of the input trace is performed, and the algorithm outputs a stream valuation of $\varphi$. We claim that the uniqueness conditions in the second phase of the algorithm are satisfied, and the output is the unique valuation model of the well-defined BSRV $\varphi$ associated with the input $\sigma_{X}$. By Proposition $2, \mathcal{A}_{\varphi}$ is universal. Thus, by the classical construction of the DFA associated with $\mathcal{A}_{\varphi}$, it holds that for all $1 \leq i \leq\left|\sigma_{X}\right|$,

(*) $\Lambda(i) \neq \emptyset$ and for each state $q$ of $\mathcal{A}_{\varphi}, q \in \Lambda(i)$ if and only if there is a run of $\mathcal{A}_{\varphi}$ over $\sigma_{X}(1), \ldots, \sigma_{X}(i)$ leading to state $q$.

Assume that the uniqueness conditions are not satisfied, and derive a contradiction. Let $i$ be the greatest position along $\sigma_{X}$ such that the uniqueness condition at step $i$ is not satisfied. Assume that $i<\left|\sigma_{X}\right|$ (the other case being simpler). For each $q \in Q$, let $\mathcal{A}_{\varphi}^{q}$ be the NFA obtained from $\mathcal{A}_{\varphi}$ by replacing the initial state $q_{0}$ with $q$. Then, by construction of the algorithm, there are a state $p \in \Lambda(i+1)$ and a run of $\mathcal{A}_{\varphi}^{p}$ over $\sigma_{X}(i+2), \ldots, \sigma_{X}\left(\left|\sigma_{X}\right|\right)$ leading to an accepting state $q_{a c c}$. By Condition $\left(^{*}\right)$ above, $\Lambda(i) \neq \emptyset$. Hence, by hypothesis, there are two distinct states $q, q^{\prime} \in \Lambda(i)$ such that $p \in \delta\left(q, \sigma_{X}(i+1)\right)$ and $p \in \delta\left(q^{\prime}, \sigma_{X}(i+1)\right)$. By Condition (*) above, we deduce that there are two distinct accepting runs of $\mathcal{A}_{\varphi}$ over $\sigma_{X}$. This is a contradiction because by Proposition $2, \mathcal{A}_{\varphi}$ is unambiguous. Hence, the uniqueness conditions in the algorithm are satisfied. Moreover, by construction, it follows that the sequence of states computed by the algorithm in the second phase is the unique accepting run $\pi$ of $\mathcal{A}_{\varphi}$ over $\sigma_{X}$. Therefore, the 
algorithm outputs the valuation model of $\varphi$ encoded by $\pi$, which is the unique valuation model of $\varphi$ associated with the input $\sigma_{X}$. Thus, since the size of the NFA $\mathcal{A}_{\varphi}$ is singly exponential in the size of $\varphi$, Theorem 5 follows.

\section{B.2 Proof of Theorem 6}

First, we recall the notion of well-formedness [8]. Given a general SRV $\varphi$ over $X$ and $Y$, the dependency graph $G_{\varphi}$ of $\varphi$ is the finite weighted directed graph whose set of vertices is $Y$ and whose set of weighted edges is defined as follows: $y \stackrel{k}{\longrightarrow} z$ is an edge of the graph iff for the equation $y=\mathrm{E}$ of $\varphi$ associated with $y$, either $k=0$ and $z$ occurs in $\mathrm{E}$, or $k \neq 0$ and $z[k \mid d]$ occurs in $\mathrm{E}$ for some $d$. The weight of a finite path of $G_{\varphi}$ is the sum of the weights of its edges. An SRV $\varphi$ is well-formed if its dependency graph $G_{\varphi}$ has no cycle with weight zero. As shown in [8], well-formedness can be checked in polynomial time and strictly implies well-definedness. Moreover, for a well-formed SRV $\varphi$, a strongly connected component $(S C C)$ of $G_{\varphi}$ can be classified as positive or negative, where an $S C C$ is positive (resp., negative) if each cycle in the $S C C$ has weight strictly positive (resp., strictly negative). The alternation depth $\operatorname{ad}(\varphi)$ of a wellformed SRV is then defined as the maximum over the number of alternations of positive and negative vertices along a path of $G_{\varphi}$, where a vertex is positive (resp., negative) if it belongs to a positive (resp., negative) $S C C$.

Theorem 6. Given a well-defined BSRV $\varphi$, one can build in doubly exponential time a $\varphi$-equivalent BSRV which is well-formed and has alternation depth 1 .

Proof. Let $\varphi$ be a well-defined BSRV over $X$ and $Y$, and $\mathcal{A}_{\varphi}=\left\langle Q, q_{0}, \delta, F\right\rangle$ be the NFA over $2^{X}$ accepting $\mathcal{L}(\varphi)$ of Theorem 1 . We denote by $\mathcal{D}_{\varphi}$ the DFA accepting $\mathcal{L}(\varphi)$ resulting from $\mathcal{A}_{\varphi}$ by the classical power set construction. Recall that $\mathcal{D}_{\varphi}=\left\langle 2^{Q},\left\{q_{0}\right\}, \delta_{\mathcal{D}}, F_{\mathcal{D}}\right\rangle$, where $F_{\mathcal{D}}=\left\{P \in 2^{Q} \mid P \cap F \neq \emptyset\right\}$ and for all $P \in 2^{Q}$ and $\iota \in 2^{X}, \delta_{\mathcal{D}}(P, \iota)=\{q \in Q \mid q \in \delta(p, \iota)$ for some $p \in P\}$. For each output variable $y \in Y$, we denote by $Q(y)$ the set of $\mathcal{A}_{\varphi}$-states $q=$ $\left(a_{-b(\varphi)}, \ldots, a_{-1}, a_{0}, a_{1}, \ldots, a_{f(\varphi)}\right) \in Q \backslash\left\{q_{0}\right\}$ such that $y$ is in the main value $a_{0}$ of $q$. In the following, we construct a $\operatorname{BSRV} \varphi^{\prime}$ over $X$ and $Y^{\prime} \supseteq Y$ satisfying the theorem, where the set of output variables $Y^{\prime}$ is defined as follows

$$
Y^{\prime}=Y \cup \bigcup_{q \in Q \backslash\left\{q_{0}\right\}}\{\mathrm{q}\} \cup \bigcup_{P \in 2^{Q}}\{\mathrm{p}\}
$$

Thus, we associate to each non-initial $\mathcal{A}_{\varphi}$-state $q$, an output variable q, and to each $\mathcal{D}_{\varphi}$-state $P \in 2^{Q}$, an output variable $\mathrm{P}$. For each $\iota \in 2^{X}$, let $\mathrm{E}_{\iota}$ be the boolean expression over $X$ encoding the input symbol $\iota$ given by $\mathrm{E}_{\iota}:=$ $\left(\bigwedge_{x \in \iota} x\right) \wedge\left(\bigwedge_{x \in X \backslash \iota} \neg x\right)$. Additionally, for all $q \in Q$ and $P \in 2^{Q}$, let $\operatorname{Acc}_{q}$ and Init $(P, \iota)$ be the boolean constants defined as follows: (i) $\operatorname{Acc}_{q}:=1$ if $q \in F$, and $\operatorname{Acc}_{q}:=0$ otherwise, and (ii) $\operatorname{Init}(P, \iota)=1$ if $P=\delta_{\mathcal{D}}\left(\left\{q_{0}\right\}, \iota\right)$, and $\operatorname{Init}(P, \iota)=0$ otherwise. Then, for all $P \in 2^{Q}, q \in Q \backslash\left\{q_{0}\right\}$, and $y \in Y$, the equations of the 
BSRV $\varphi^{\prime}$ for the output variables $\mathrm{P}, \mathbf{q}$, and $y$ are defined as follows.

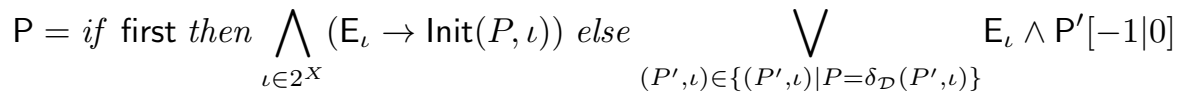

$$
\begin{aligned}
& \mathrm{q}=\text { if } \bigvee_{P \in\left\{P \in 2^{Q} \mid q \in P\right\}} \mathrm{P} \text { then } \mathrm{E}_{q} \text { else } 0 \\
& \text { where } \mathrm{E}_{q}:=\text { if last then } \mathrm{Acc}_{q} \text { else } \bigvee_{\iota \in 2^{X}} \bigvee_{q^{\prime} \in \delta(q, \iota)}\left(\mathrm{E}_{\iota}[+1 \mid 0] \wedge \mathrm{q}^{\prime}[+1 \mid 0]\right) \\
& y=\bigvee_{q \in Q(y)} \mathrm{q}
\end{aligned}
$$

By construction, it easily follows that the BSRV $\varphi^{\prime}$ is well-formed (hence, welldefined too) and the alternation depth of $\varphi^{\prime}$ is exactly 1. It remains to show that $\varphi^{\prime}$ is $\varphi$-equivalent. Let $\sigma_{X}$ be an input stream valuation, and $\sigma$ and $\sigma^{\prime}$ be the unique valuation models of $\varphi$ and $\varphi^{\prime}$, respectively, associated with the input $\sigma_{X}$. We need to prove that the restrictions of $\sigma$ and $\sigma^{\prime}$ to $Y$ coincide. Since $\varphi$ is well-defined, by Proposition $2, \mathcal{A}_{\varphi}$ is universal and unambiguous. Let $\pi=q_{0}, q_{1}, \ldots, q_{\left|\sigma_{X}\right|}$ be the unique accepting run of $\mathcal{A}_{\varphi}$ over $\sigma_{X}$ (which encodes $\sigma)$. Then, by the equations of $\varphi^{\prime}$ associated with the output variables $y \in Y$, it suffices to prove the following condition $(*)$ : for each $1 \leq i \leq\left|\sigma_{X}\right|$, there is exactly one state $p \in Q \backslash\left\{q_{0}\right\}$ such that $\mathrm{p} \in \sigma^{\prime}(i)$ (i.e., such that the boolean value of $\mathrm{p}$ at position $i$ with respect to $\sigma^{\prime}$ is 1$)$; moreover, $p=q_{i}$. Let $\pi_{\mathcal{D}}=\left\{q_{0}\right\}, P_{1}, \ldots, P_{\left|\sigma_{X}\right|}$ be the run of $\mathcal{D}_{\varphi}$ over $\sigma_{X}$. First, we observe that the equations for the output variables $\mathrm{P}$ ensure that for each $1 \leq i \leq\left|\sigma_{X}\right|$, there is exactly one $\mathcal{D}_{\varphi}$-state $P \in 2^{Q}$ such that $\mathrm{P} \in \sigma^{\prime}(i)$; moreover, $P=P_{i}$. By using this observation and the fact that $\mathcal{A}_{\varphi}$ is universal and unambiguous, and proceeding as in the proof of correctness of the algorithm of Figure 4, Condition $\left(^{*}\right)$ easily follows, which concludes.

\section{Proofs from Section 5}

\section{C.1 Proof of Proposition 3}

The main result of Section 5 is Theorem 7 .

Theorem 7. For BSRV:

1. The under-definedness problem is PSPACE-complete, the well-definedness problem is in EXPTIME and at least PSPACE-hard, while the over-definedness problem and the generalized well-definedness problem are both EXPSPACEcomplete.

2. Checking semantic equivalence is EXPSPACE-complete.

3. When interpreted as language recognizers, language emptiness is PSPACEcomplete, while language universality, language inclusion, and language equivalence are EXPSPACE-complete. 
The upper-bounds of Theorem 7(1) where shown in Section 5. The lowerbounds are proved in Theorems 8 and 9 below. Theorem 10 corresponds to Theorem 7(3), and Theorem 11 corresponds to Theorem 7(2).

First, we include the full proof of the auxiliary result, Proposition 3.

Proposition 3. Given an NFA $\mathcal{A}$ and a state projection $\Upsilon$ of $\mathcal{A}$, checking whether $\mathcal{A}$ is not unambiguous with respect to $\Upsilon$ can be done in NLOGSPACE.

Proof. The nondeterministic algorithm solving the considered problem, given the input $(\mathcal{A}, \Upsilon)$, proceeds as follows: at each step, the algorithm guesses two runs $\pi$ and $\pi^{\prime}$ of $\mathcal{A}$ over the same input. The algorithm keeps in memory only the pair of states $\left(q, q^{\prime}\right)$, where $q$ (resp. $q^{\prime}$ ) is the last state of $\pi$ (resp., $\pi^{\prime}$ ), and a flag $f$ which is 1 iff the projections $\Upsilon(\pi)$ and $\Upsilon\left(\pi^{\prime}\right)$ of the two runs $\pi$ and $\pi^{\prime}$ guessed so far are distinct (initially, $q$ and $q^{\prime}$ coincide with the initial state, and $f=0$ ). If $f=1$ and $q$ and $q^{\prime}$ are both accepting (hence, $\pi$ and $\pi^{\prime}$ are two accepting runs over the same input and $\Upsilon(\pi)$ and $\Upsilon\left(\pi^{\prime}\right)$ are distinct), then the algorithm terminates with success. Otherwise, the algorithm guesses two transitions of $\mathcal{A}$ from $q$ and $q^{\prime}$ reading the same input symbol, leading to states $p$ and $p^{\prime}$, respectively, re-writes the memory by replacing the pair $\left(q, q^{\prime}\right)$ with the new pair $\left(p, p^{\prime}\right)$, and the flag $f$ with the new flag $f^{\prime}$, where $f^{\prime}$ is 1 iff either $f=1$ or $\Upsilon(p)$ and $\Upsilon\left(p^{\prime}\right)$ are distinct, and the whole procedure is repeated.

Theorem 8. The over-definedness and generalized well-definedness problems for BSRV are both EXPSPACE-hard.

Proof. First, we observe that the complement of the over-definedness problem can be reduced in linear time to the generalized well-definedness problem. Indeed, let $\varphi$ be a BSRV and $\varphi^{\prime}$ be the BSRV obtained from $\varphi$ by adding the equation $z=0$, where $z$ is a fresh output variable. Evidently, $\varphi$ is not over-defined iff $\varphi^{\prime}$ is well-defined with respect to $\{z\}$. Hence, it suffices to prove EXPSPACE-hardness for the over-definedness problem. The result is obtained by a polynomial-time reduction from a domino-tiling problem for grids with rows of singly exponential length [14]. An instance $\mathcal{I}$ of this problem is a tuple $\mathcal{I}=\left\langle C, \Delta, m, d_{\text {init }}, d_{\text {final }}\right\rangle$, where $C$ is a finite set of colors, $\Delta \subseteq C^{4}$ is a set of tuples $\left\langle c_{\text {down }}, c_{\text {left }}, c_{\text {up }}, c_{\text {right }}\right\rangle$ of four colors, called domino-types, $m>0$ is a natural number (written in unary), and $d_{\text {init }}, d_{\text {final }} \in \Delta$ are two domino-types. For $n>0$, a tiling of $\mathcal{I}$ for the $n \times 2^{m}$-grid is a mapping $f:[0, n-1] \times\left[0,2^{m}-1\right] \rightarrow \Delta$ satisfying the following:

- two adjacent cells in a row have the same color on the shared edge: for all $(i, j) \in[0, n-1] \times\left[0,2^{m}-1\right]$ with $j<2^{m}-1,[f(i, j)]_{\text {right }}=[f(i, j+1)]_{\text {left }}$;

- two adjacent cells in a column have the same color on the shared edge: for all $(i, j) \in[0, n-1] \times\left[0,2^{m}-1\right]$ with $i<n-1,[f(i, j)]_{\text {up }}=[f(i+1, j)]_{\text {down }}$; $-f(0,0)=d_{\text {init }}$ and $f\left(n-1,2^{m}-1\right)=d_{\text {final }}$.

A tiling of $\mathcal{I}$ is a tiling of $\mathcal{I}$ for the $n \times 2^{m}$-grid for some $n>0$. It is well-known that checking the existence of a tiling for $\mathcal{I}$ is EXPSPACE-complete [14]. In the 
following, we construct in polynomial-time a BSRV $\varphi$ such that there exists a tiling for $\mathcal{I}$ iff $\varphi$ is over-defined. The set $X$ of input variables of $\varphi$ is given by

$$
X:=\{\mathrm{d} \mid d \in \Delta\} \cup\left\{\mathrm{b}_{1}^{+}, \ldots, \mathrm{b}_{\mathrm{m}}^{+}, \mathrm{b}_{1}^{-}, \ldots, \mathrm{b}_{\mathrm{m}}^{-}\right\}
$$

Thus, we associate to each domino-type $d \in \Delta$ an input variable $\mathrm{d}$. Moreover, the additional input variables $b_{1}^{+}, \ldots, b_{m}^{+}, b_{1}^{-}, \ldots, b_{m}^{-}$are used to encode the value of a $m$-bits counter numbering the cells of one row of the grid $\left(b_{1}^{+}\right.$is 1 iff the $i$ th bit is 1 , and $b_{i}^{-}$is 1 iff the $i$ th bit is 0 ). Thus, a cell is encoded as a sequence of length $m+1$, the first $m$ positions giving the binary encoding of the column number and the last position giving the associated domino-type. ${ }^{1}$ Moreover, a tiling is encoded as a sequence of rows, starting from the first row, and a row lists the (encodings of) cells from left to right.

In the following, for a stream variable $y$ and an integer $k$, we use $y[k]$ for the stream expression $y[k \mid 1]$ if $k \neq 0$, and for the stream expression $y$ otherwise.

Now, we illustrate the construction of $\varphi$ ensuring that the unique inputs for which there is no output stream valuation are those encoding tilings of $\mathcal{I}$. We use a uniform output variable PTU in order to check that the input encodes a pseudo tiling, i.e. a tiling of $\mathcal{I}$, where the requirement that two adjacent cells in a column have the same color on the shared edge is relaxed. For this, we use an additional output variable PT which assumes the value 1 everywhere iff the input streams encode a pseudo tiling. The equation for variable PT is as follows.

$$
\begin{aligned}
& \mathrm{PT}=\underbrace{\bigvee_{x \in X}\left(x \wedge \bigwedge_{x^{\prime} \in X \backslash\{x\}} \neg x^{\prime}\right)}_{\text {exactly one input variable has value } 1} \wedge \\
& \underbrace{\bigwedge_{i=1}^{m-1}\left(\left(\mathrm{~b}_{\mathrm{i}}^{+} \vee \mathrm{b}_{\mathrm{i}}^{-}\right) \rightarrow\left(\neg \text { last } \wedge\left(\mathrm{b}_{\mathrm{i}+1}^{+}[+1] \vee \mathrm{b}_{\mathrm{i}+1}^{-}[+1]\right)\right)\right) \wedge\left(\left(\mathrm{b}_{\mathrm{m}}^{+} \vee \mathrm{b}_{\mathrm{m}}^{-}\right) \rightarrow\left(\neg \text { last } \wedge \bigvee_{d \in \Delta} \mathrm{d}[+1]\right)\right)} \wedge \\
& \text { the input is a list of numbered cells }
\end{aligned}
$$

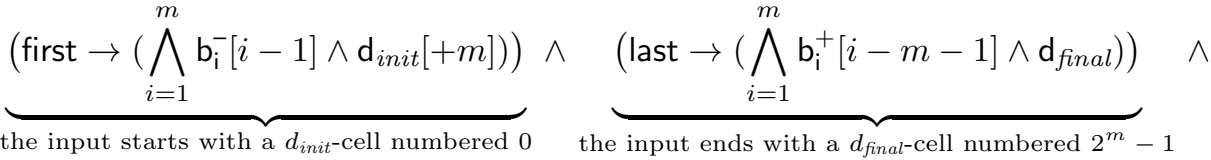

$$
\begin{aligned}
& \left\{( \neg \text { last } \wedge \bigvee _ { d \in \Delta } \mathrm { d } ) \longrightarrow \left(\left(\mathrm{b}_{1}^{+}[+1] \vee \mathrm{b}_{1}^{-}[+1]\right) \wedge\right.\right. \\
& \underbrace{\left.\left.\left(\mathrm{b}_{1}^{+}[-m] \leftrightarrow \mathrm{b}_{1}^{-}[+1]\right) \wedge \bigwedge_{i=1}^{m-1}\left(\mathrm{~b}_{\mathrm{i}+1}^{+}[-m+i] \leftrightarrow \mathrm{b}_{\mathrm{i}+1}^{-}[i+1]\right) \leftrightarrow\left(\mathrm{b}_{\mathrm{i}}^{+}[-m+i-1] \wedge \mathrm{b}_{\mathrm{i}}^{-}[i]\right)\right)\right\}}_{\text {the cells are listed in }} \wedge \\
& \bigwedge_{d \in \Delta} \mathrm{d} \rightarrow\left(\text { last } \vee \bigwedge_{i=1}^{i=m} \mathrm{~b}_{\mathrm{i}}^{-}[i] \vee \bigvee_{d^{\prime} \in \Delta:\left(d^{\prime}\right)_{\text {left }}=(d)_{\text {right }}} \mathrm{d}^{\prime}[m+1]\right)
\end{aligned}
$$

\footnotetext{
${ }^{1}$ We assume that the first bit is the least significant one.
} 
The equation for the uniform output variable PTU is as follows. We also use an extra variable test ${ }_{1}$ in order to avoid situations where PT is everywhere 1 and the uniform value of PTU is 0 .

$$
\begin{aligned}
& \mathrm{PTU}=\text { if }(\neg \text { first } \rightarrow(\mathrm{PTU}[-1] \leftrightarrow \mathrm{PTU})) \wedge(\neg \mathrm{PT} \rightarrow \neg \mathrm{PTU}) \text { then } \mathrm{PTU} \text { else } \neg \mathrm{PTU} \\
& \text { test }_{1}=\text { if }\left\{\left((\text { first } \wedge \mathrm{PT} \wedge \neg \mathrm{PTU}) \rightarrow \neg \text { test }_{1}\right) \wedge\left(\text { last } \rightarrow \text { test }_{1}\right) \wedge\right. \\
& \left.\left(\left(\neg \text { first } \wedge \neg \text { test }_{1}[-1] \wedge \mathrm{PT} \wedge \neg \mathrm{PTU}\right) \rightarrow \neg \text { test }_{1}\right)\right\} \text { then } \text { test }_{1} \text { else } \rightarrow \text { test }_{1}
\end{aligned}
$$

Thus, the uniform value of PTU is 1 iff the input encodes a pseudo tiling. Now, we describe the crucial step of the construction of $\varphi$. Assume that the input encodes a pseudo tiling, i.e. the uniform value of PTU is 1 . Then, the input is not a tiling of $\mathcal{I}$ iff there are two adjacent cells $(i, j)$ and $(i+1, j)$ in some column which have different color on the shared edge. In order to check this last condition, we use $O(m+|\Delta|)$ additional output variables. In particular, for each $h=1,2$, we use the output variable $\mathrm{Bl}_{\mathrm{h}}$ for marking a cell of the pseudo tiling: if the uniform value of PTU is 1 , then the stream for $B I_{h}$ is in $0^{*} 1^{+}$and the suffix in $1^{+}$starts with a cell ("the cell marked by $\mathrm{Bl}_{\mathrm{h}}$ ").

$$
\begin{aligned}
\mathrm{Bl}= & \text { if } \mathrm{PTU} \rightarrow\left\{\left(\text { last } \rightarrow \mathrm{Bl}_{\mathrm{h}}\right) \wedge\left(\left(\mathrm{Bl}_{\mathrm{h}} \wedge \neg \mathrm{Bl}_{\mathrm{h}}[-1]\right) \rightarrow\left(\mathrm{b}_{1}^{+} \vee \mathrm{b}_{1}^{-}\right)\right) \wedge\right. \\
& \left.\left(\left.\neg \mathrm{B}\right|_{\mathrm{h}} \rightarrow \neg \mathrm{Bl}_{\mathrm{h}}[-1 \mid 0]\right) \wedge\left(\mathrm{Bl}_{\mathrm{h}} \rightarrow \mathrm{Bl}_{\mathrm{h}}[+1]\right)\right\} \text { then } \mathrm{Bl}_{\mathrm{h}} \text { else } \neg \mathrm{Bl}_{\mathrm{h}}
\end{aligned}
$$

Moreover, we use the output variables $\mathrm{m}_{1}, \mathrm{~m}_{2}$, and test $\mathrm{t}_{2}$ in order to ensure that the cells marked by $\mathrm{Bl}_{1}$ and $\mathrm{Bl}_{2}$ belongs to two adjacent rows (with the $\mathrm{Bl}_{2}$ 's row following the $\mathrm{Bl}_{1}$ 's row). The stream for $\mathrm{m}_{1}$ is in $0^{*} 1^{+}$and the suffix in $1^{+}$starts with the first cell numbered 0 following the cell marked by $\mathrm{Bl}_{1}\left(\mathrm{Bl}_{1}\right.$ included), while the stream for $\mathrm{m}_{2}$ is in $1^{+} 0^{+}$and the prefix in $1^{+}$ends with the first bit of the last cell numbered 0 which precedes the cell marked by $\mathrm{Bl}_{2}\left(\mathrm{Bl}_{2}\right.$ excluded). Finally, the variable test ${ }_{2}$ is used to check that the first 1-value bit of $\mathrm{m}_{1}$ corresponds to the last 1-value bit of $\mathrm{m}_{2}$.

$$
\begin{aligned}
& \mathrm{m}_{1}=\text { if } \mathrm{PTU} \rightarrow\left\{\left(\text { last } \rightarrow \mathrm{m}_{1}\right) \wedge\left(\neg \mathrm{m}_{1} \rightarrow \neg \mathrm{m}_{1}[-1 \mid 0]\right) \wedge\right. \\
&\left(\mathrm{m}_{1} \rightarrow \mathrm{m}_{1}[+1]\right) \wedge\left(\left(\mathrm{Bl}_{1} \wedge \bigwedge_{i=1}^{m} \mathrm{~b}_{\mathrm{i}}^{-}[i-1]\right) \rightarrow \mathrm{m}_{1}\right) \wedge \\
&\left.\left(\left(\mathrm{m}_{1} \wedge \neg \mathrm{m}_{1}[-1 \mid 0]\right) \rightarrow\left(\mathrm{Bl}_{1} \wedge \bigwedge_{i=1}^{m} \mathrm{~b}_{\mathrm{i}}^{-}[i-1]\right)\right)\right\} \text { then } \mathrm{m}_{1} \text { else } \neg \mathrm{m}_{1} \\
& \mathrm{~m}_{2}=\text { if } \mathrm{PTU} \rightarrow\left\{\left(\text { last } \rightarrow \neg \mathrm{m}_{2}\right) \wedge\left(\text { first } \rightarrow \mathrm{m}_{2}\right) \wedge\left(\mathrm{m}_{2} \rightarrow \mathrm{m}_{2}[-1]\right) \wedge\right. \\
&\left(\neg \mathrm{m}_{2} \rightarrow \neg \mathrm{m}_{2}[+1 \mid 0]\right) \wedge\left(\left(\neg \mathrm{Bl}_{2} \wedge \bigwedge_{i=1}^{m} \mathrm{~b}_{\mathrm{i}}^{-}[i-1]\right) \rightarrow \mathrm{m}_{2}\right) \wedge \\
&\left.\left(\left(\mathrm{m}_{2} \wedge \neg \mathrm{m}_{2}[+1]\right) \rightarrow\left(\neg \mathrm{BI}_{2} \wedge \bigwedge_{i=1}^{m} \mathrm{~b}_{\mathrm{i}}^{-}[i-1]\right)\right)\right\} \text { then } \mathrm{m}_{2} \text { else } \neg \mathrm{m}_{2} \\
& \text { test } \mathrm{m}_{2}=\text { if } \text { PTU } \rightarrow\left\{\left(\mathrm{m}_{2} \wedge \neg \mathrm{m}_{2}[+1]\right) \leftrightarrow\left(\mathrm{m}_{1} \wedge \neg \mathrm{m}_{1}[-1 \mid 0]\right)\right\} \\
& \text { then test } \mathrm{m}_{2} \text { else } \neg \text { test }_{2}
\end{aligned}
$$

Finally, we use the output variables ob $1, \ldots$, ob $_{\mathrm{m}}$, test 3 , and od for each $d \in \Delta$, for ensuring that the cells marked by $\mathrm{Bl}_{1}$ and $\mathrm{Bl}_{2}$ have the same number (i.e., belong to the same column) and have different color on the shared edge. The 
equation for $\mathrm{ob}_{\mathrm{i}}$ ensures that there is a uniform stream for $\mathrm{ob}_{\mathrm{i}}$ iff the $i$ th bits of the cells marked by $\mathrm{Bl}_{1}$ and $\mathrm{Bl}_{2}$ have the same value. Moreover, for each $d \in \Delta$, the equation for od ensures that there is a uniform stream for od iff either the domino-type of the cell marked by $\mathrm{Bl}_{1}$ is not $d$, or the cells marked by $\mathrm{Bl}_{1}$ and $\mathrm{Bl}_{2}$ have different color on the shared edge. Thus, the equation for variable test 3 requires that the output streams for $\mathrm{ob}_{1}, \ldots, \mathrm{ob}_{\mathrm{m}}$, od $(d \in \Delta)$ are uniform.

$$
\begin{aligned}
& \mathrm{ob}_{\mathrm{i}}=\text { if } \mathrm{PTU} \rightarrow \bigwedge_{h=1}^{2}\left\{\left(\mathrm{Bl}_{\mathrm{h}} \wedge \neg \mathrm{Bl}_{\mathrm{h}}[-1 \mid 0]\right) \longrightarrow\right. \\
& \left.\left(\left(\mathrm{b}_{\mathrm{i}}^{+}[i-1] \rightarrow \mathrm{ob}_{\mathrm{i}}[i-1]\right) \wedge\left(\mathrm{b}_{\mathrm{i}}^{-}[i-1] \rightarrow \neg \mathrm{ob}_{\mathrm{i}}[i-1]\right)\right)\right\} \text { then } \mathrm{ob}_{\mathrm{i}} \text { else } \neg \mathrm{ob}_{\mathrm{i}} \\
& \text { od }=\text { if } \mathrm{PTU} \rightarrow\left\{\left(\left(\mathrm{d}[m] \wedge \mathrm{Bl}_{1} \wedge \neg \mathrm{BI}_{1}[-1 \mid 0]\right) \rightarrow \operatorname{od}[m]\right) \wedge\right. \\
& \left.\left(\left(\bigvee_{d^{\prime} \in \Delta:\left(d^{\prime}\right)_{\text {down }}=(d)_{\text {up }}} \mathrm{d}^{\prime}[m] \wedge \mathrm{BI}_{2} \wedge \neg \mathrm{BI}_{2}[-1]\right) \rightarrow \neg \mathrm{od}[m]\right)\right\} \text { then od else } \neg \text { od } \\
& \text { test }_{3}=\text { if PTU } \rightarrow \neg \text { first } \rightarrow\left(\bigwedge_{i=1}^{m}\left(\mathrm{ob}_{\mathrm{i}}[-1] \leftrightarrow \mathrm{ob}_{\mathrm{i}}\right) \wedge \bigwedge_{d \in \Delta}(\operatorname{od}[-1] \leftrightarrow \text { od })\right) \\
& \text { then } \text { test }_{3} \text { else } \neg \text { test }_{3}
\end{aligned}
$$

Hence, the unique inputs for which the constructed BSRV $\varphi$ has no output stream valuation are those encoding tilings of $\mathcal{I}$. Thus, $\varphi$ is over-defined iff there is a tiling of $\mathcal{I}$. Note that the size of $\varphi$ is quadratic in the size of $\mathcal{I}$. This concludes the proof of Theorem 8 .

Theorem 9. The under-definedness and well-definedness problems for BSRV are both PSPACE-hard.

Proof. By a polynomial-time reduction from a domino-tiling problem for grids with rows of polynomial length [14]. An instance $\mathcal{I}=\left\langle C, \Delta, m, d_{\text {init }}, d_{\text {final }}\right\rangle$ of this problem is defined as in the proof of Theorem 8 . However, here, a tiling of $\mathcal{I}$ is a tiling of $\mathcal{I}$ for the $n \times m$-grid for some $n>0$ (i.e., the length of any row is $m$ ). It is well-known that checking the existence of a tiling for $\mathcal{I}$ is PSPACEcomplete [14]. We construct in polynomial time a BSRV $\varphi$ such that the following holds:

- there exists a tiling of $\mathcal{I}$ iff $\varphi$ is under-defined;

$-\varphi$ is not under-defined iff $\varphi$ is well-defined.

Hence, the result follows. Now, we illustrate the construction of $\varphi$. The set $X$ of input variables of the specification $\varphi$ is given by

$$
X:=\{\mathrm{d} \mid d \in \Delta\} \cup\{\mathrm{mk}\}
$$

Thus, we associate to each domino-type $d \in \Delta$ an input variable $\mathrm{d}$. Moreover, the additional input variable $\mathrm{mk}$ is used as a separator between two adjacent rows. Thus, a tiling is encoded as a sequence of rows separated by the special marker, starting from the first row. Additionally, the first row is preceded by the special marker, and the last row is followed by the special marker. The specification $\varphi$ has two output variables: PT and PTU. The output variable PT is used to check that the input encodes a tiling of $\mathcal{I}$ : in particular, PT assumes the value 
1 everywhere iff the input streams encode a tiling. Formally, the equation for variable PT is as follows.

$$
\begin{aligned}
& \mathrm{PT}=\underbrace{\bigvee_{x \in X}\left(x \wedge \bigwedge_{x^{\prime} \in X \backslash\{x\}} \neg x^{\prime}\right)}_{\text {exactly one input variable has value } 1} \wedge \\
& \underbrace{\left(\text { first } \rightarrow\left(\mathrm{mk} \wedge \mathrm{d}_{\text {init }}[+1 \mid 0]\right)\right)}_{\text {the input starts with a } d_{\text {init }} \text {-cell }} \wedge \underbrace{\left(\text { last } \rightarrow\left(\mathrm{mk} \wedge \mathrm{d}_{\text {final }}[-1 \mid 0]\right)\right)}_{\text {the input ends with a } d_{\text {final }} \text {-cell }} \wedge \\
& \underbrace{\left((\mathrm{mk} \wedge \neg \text { last }) \longrightarrow\left(\mathrm{mk}[m+1 \mid 0] \wedge \bigwedge_{i=1}^{m} \neg \mathrm{mk}[i \mid 1]\right)\right)} \wedge \\
& \text { adjacent rows are separated by the marker } \\
& \underbrace{\bigwedge_{d \in \Delta}\left(\mathrm{d} \rightarrow\left(\mathrm{mk}[+1 \mid 0] \vee \bigvee_{d^{\prime} \in \Delta:\left(d^{\prime}\right)_{\text {left }}=(d)_{\text {right }}} \mathrm{d}^{\prime}[+1 \mid 0]\right)\right) \wedge}_{\text {two adjacent cells in a row have the same color on the shared edge }} \\
& \underbrace{\bigwedge_{d \in \Delta}\left(\mathrm{d} \rightarrow \bigvee_{d^{\prime} \in \Delta:\left(d^{\prime}\right)_{\text {down }}=(d)_{u p}} \mathrm{~d}^{\prime}[m+1 \mid 1]\right)}_{\text {two adjacent cells in a column have the same color on the shared edge }}
\end{aligned}
$$

Finally, the equation for the uniform output variable PTU is as follows.

$$
\mathrm{PTU}=\text { if }(\neg \text { first } \rightarrow(\mathrm{PTU}[-1 \mid 1] \leftrightarrow \mathrm{PTU})) \wedge(\neg \mathrm{PT} \rightarrow \neg \mathrm{PTU}) \text { then } \mathrm{PTU} \text { else } \neg \mathrm{PTU}
$$

Note that if the input does not encode a tiling (i.e., for some position, PT assumes the value 0 ), then the uniform value of PTU is 0 . Otherwise, the uniform value of PTU may be 0 or 1 . Since for each input, the stream valuation for the other output variable PT is uniquely determined, it follows that there is a tiling for $\mathcal{I}$ iff $\varphi$ is under-defined. Moreover, since for each input, there is some output stream valuation, it follows that $\varphi$ is not under-defined iff $\varphi$ is well-defined. Note that the size of $\varphi$ is quadratic in the size of $\mathcal{I}$. Hence, the result follows, which concludes the proof of Theorem 9 .

Theorem 10. For BSRV, viewed as language recognizers, language emptiness is PSPACE-complete, while language universality, language inclusion, and language equivalence are EXPSPACE-complete.

Proof. Recall that for NFA, emptiness is NLOGSPACE-complete, while universality, inclusion, and equivalence are PSPACE-complete. Hence, the upper bounds of the theorem directly follows from Theorem 1. The matching lower bounds for universality, inclusion, and equivalence directly follows from Theorem 8 and the facts that language universality for BSRV is the complement of the overdefinedness problem, and language inclusion and language equivalence can be reduced in linear time to language universality. It remains to show that language emptiness for BSRV is PSPACE-hard. We modify the polynomial-time reduction 
given in the proof of Theorem 9 as follows: the equation for the output variable PTU of the BSRV $\varphi$ is updated as follows:

$$
\mathrm{PTU}=\text { if }(1 \leftrightarrow \mathrm{PTU}) \wedge(\neg \mathrm{PT} \rightarrow \neg \mathrm{PTU}) \text { then } \mathrm{PTU} \text { else } \neg \mathrm{PTU}
$$

Hence, PTU is a uniform output variable whose uniform value is always 1. Moreover, the output stream for PTU is defined iff the output stream for PT is in $1^{+}$. Additionally, the construction in the proof of Theorem 9 ensures that for each input, the output stream for the output variable PT is uniquely determined, and PT assumes the value 1 everywhere iff the input streams encode a tiling. Hence, the updated construction is a polynomial-time reduction from a PSPACEcomplete problem to language emptiness for BSRV, and we are done.

Theorem 11. Checking semantic equivalence for BSRV is EXPSPACE-complete.

Proof. Let $\varphi$ be a BSRV over $X$ and $Y, \varphi^{\prime}$ be a BSRV over $X$ and $Y^{\prime}$, and $Z \subseteq Y \cap Y^{\prime}$. Note that if $Z=\emptyset$, then $\varphi$ and $\varphi^{\prime}$ are equivalent with respect to $Z$ iff $\mathcal{L}(\varphi)=\mathcal{L}\left(\varphi^{\prime}\right)$. Hence, the lower bound directly follows from Theorem 10 . Now, let us consider the upper bound. By a straightforward adaptation of Theorem 1, one can construct in singly exponential time an NFA $\mathcal{A}_{\varphi}$ (resp., $\mathcal{A}_{\varphi^{\prime}}$ ) over $2^{X \cup Z}$ such that $\mathcal{L}\left(\mathcal{A}_{\varphi}\right)$ (resp., $\mathcal{L}\left(\mathcal{A}_{\varphi^{\prime}}\right)$ ) is the set of stream valuations over $X \cup Z$ which can be extended to valuation models of $\varphi\left(\right.$ resp., $\left.\varphi^{\prime}\right)$. It follows that $\varphi$ and $\varphi^{\prime}$ are equivalent with respect to $Z$ iff $\mathcal{L}\left(\mathcal{A}_{\varphi}\right)=\mathcal{L}\left(\mathcal{A}_{\varphi^{\prime}}\right)$. Thus, since language equivalence for NFA is PSPACE-complete, membership in EXPSPACE for the considered problem follows. 